\title{
- Kernel reconstruction methods for Doppler broadening - temperature interpolation by linear combination of reference cross sections at optimally chosen temperatures
}

\author{
Pablo Ducru ${ }^{\mathrm{a}}$, Colin Josey ${ }^{\mathrm{a}}$, Karia Dibert ${ }^{\mathrm{a}}$, Vladimir Sobes ${ }^{\mathrm{b}}$, Benoit Forget $^{\mathrm{a}}$, Kord Smith $^{\mathrm{a}}$ \\ ${ }^{a}$ Nuclear Science 8$\}$ Engineering department, Massachusetts Institute of Technology, \\ 77 Massachusetts Ave., Cambridge, MA 02139, USA. \\ ${ }^{b}$ Oak Ridge National Laboratory \\ 1 Bethel Valley Rd., Oak Ridge, TN, USA.
}

\begin{abstract}
This article establishes a new family of methods to perform temperature interpolation of nuclear interactions cross sections, reaction rates, or cross sections times the energy. One of these quantities at temperature $T$ is approximated as a linear combination of quantities at reference temperatures $\left(T_{j}\right)$. The problem is formalized in a cross section independent fashion by considering the kernels of the different operators that convert cross section related quantities from a temperature $T_{0}$ to a higher temperature $T$ namely the Doppler broadening operation. Doppler broadening interpolation of nuclear cross sections is thus here performed by reconstructing the kernel of the operation at a given temperature $T$ by means of linear combination of kernels at reference temperatures $\left(T_{j}\right)$. The choice of the $\mathrm{L}_{2}$ metric yields optimal linear interpolation coefficients in the form of the solutions of a linear algebraic system inversion. The optimization of the choice of reference temperatures $\left(T_{j}\right)$ is then undertaken so as to best reconstruct, in the $\mathrm{L}_{\infty}$ sense, the kernels over a given temperature range $\left[T_{\min }, T_{\max }\right]$. The performance of these kernel reconstruction methods is then assessed in light of previous temperature interpolation methods by testing them upon isotope ${ }^{238} \mathrm{U}$. Temperature-optimized free Doppler kernel reconstruction significantly outperforms all previous interpolation-based methods, achieving $0.1 \%$ relative error on temperature interpolation of ${ }^{238} \mathrm{U}$ total cross section over the temperature range $[300 \mathrm{~K}, 3000 \mathrm{~K}]$ with only 9 reference temperatures.
\end{abstract}

Keywords: Doppler broadening, nuclear cross sections, temperature interpolation, kernel reconstruction.

\section{Introduction}

Temperature effects play a crucial role in nuclear reactor physics. One such effect - Doppler broadening - is for instance responsible for important feedback mechanisms that can ensure the stability of a critical reactor.

In both deterministic and Monte Carlo neutron transport calculations, the temperature dependence of nuclear cross sections is often dealt with by pre-tabulating $N$ Doppler-broadened cross sections, $\sigma_{T_{j}}(E)$, at a sequence of given temperatures, $\left(T_{j}\right)_{j \in \llbracket 1 ; N \rrbracket}$, and then interpolating those values through some interpolation scheme to find the cross section, $\sigma_{T}(E)$, at the desired temperature $T$ [1]. Such an interpolation is necessary in practice due to the difficulty of calculating the exact Doppler broadening on-the-fly. So far, Doppler broadening temperature interpolation has been traditionally performed by ad hoc methods that were not the result of a rigorous optimization problem, and which were selected on best-performance criteria.

Email addresses: p_ducru@mit.edu (Pablo Ducru), cjosey@mit.edu (Colin Josey), kdibert@mit.edu (Karia Dibert), sobesv@ornl.gov (Vladimir Sobes), bforget@mit.edu (Benoit Forget), kord@mit.edu (Kord Smith) 
In this article, a new family of linear interpolation methods is developed: kernel reconstruction methods. They rest on the mathematical concept of minimizing the distance between the kernels of the linear operators that transform a cross section, a reaction rate, or energy times the cross section, from one temperature to another.

As a physical interpretation, these kernel reconstructions seek to recreate the velocity (or energy) distribution of the target particle at a given temperature, $T$, with a linear combination of the distributions at the reference temperatures, $\left(T_{j}\right)$. This idea had been introduced, though not systematically formalized, in [2], for a particular case of reaction rates.

In section 2, this article presents the principle of linear kernel reconstruction methods for Doppler broadening, and presents four natural kernel reconstruction methods: Doppler kernel reconstruction; Maxwellian kernel reconstruction; Maxwellian-over-velocity kernel reconstruction; and Boltzmann kernel reconstruction. In each case, the linear reconstruction can be performed as a free optimization problem or as a constrained one, for a total of eight methods. For each method, the optimal coefficients - in the $\mathrm{L}_{2}$ norm sense - for linear interpolation are exhibited as the solution to an inversion of a cross section independent system. Thus, provided a set of reference temperatures $\left(T_{j}\right)$ and a target temperature $T$ to which to interpolate, the kernel reconstruction methods provide optimally chosen interpolation coefficients.

Section 3 further temperature-optimizes these kernel reconstruction methods by finding the set of reference temperatures $\left(T_{j}\right)_{j \in \llbracket 1 ; N \rrbracket}$ that minimize the kernel reconstruction relative error over the entire temperature range $\left[T_{\min }, T_{\max }\right]$ on which the quantities are to be interpolated. An algorithm specifically devised to solve the min-max problem of such a temperature-optimization is introduced. Each kernel reconstruction method is tested with its own optimized reference temperatures grid to assess kernel reconstruction performance.

Finally, section 4 studies the performance of the kernel reconstruction methods, with their respective optimal $\left(T_{j}\right)_{j \in \llbracket 1 ; N \rrbracket}$ reference temperature distributions, on nuclide ${ }^{238} \mathrm{U}$. Temperature-optimized constrained Doppler kernel reconstruction is shown to have the best accuracy amongst all eight kernel reconstruction methods. However, the free Doppler kernel reconstruction method was preferred due to its close accuracy and higher algorithmic and computational performance. Free Doppler kernel is then compared to previous Doppler broadening interpolation methods commonly in use in nuclear reactor physics calculations.

\section{Kernel reconstruction linear combination temperature interpolation methods}

This section defines and establishes the various kernel reconstruction methods to perform Doppler broadening temperature interpolation by means of linear combination of reference cross sections, reaction rates, or energy-times-cross sections.

\subsection{Dopper broadening operation}

Assuming Maxwell-Boltzmann's free-gas model for the target nuclei distributions of velocities $\overrightarrow{v_{t}}$ and energies, the Doppler broadening effect of temperature on nuclear reaction rates in the resolved resonance range can be modeled as follows [3]:

$$
v \sigma_{T}(v)=\int_{v_{t} \mid v_{r}>0} v_{r} \sigma_{T_{0}}\left(v_{r}\right) \mathcal{M}_{T}\left(\overrightarrow{v_{t}}\right) d \overrightarrow{v_{t}}
$$

where $T$ is the temperature of the target nuclei; $T_{0}$ a given reference temperature which will henceforth be, without loss of generality, assumed to be zero; $v_{r}$ is the relative speed between the neutron hitting at speed $v$ the target that has a Maxwell-Boltzmann velocity distribution $\mathcal{M}_{T}\left(\overrightarrow{v_{t}}\right)$; and $\sigma_{T}(v)$ designates the interaction cross section at temperature $T$ for incoming neutron speed $v$ (respectively temperature $T_{0}$ and relative speed $v_{r}$ for $\sigma_{T_{0}}\left(v_{r}\right)$ ). The assumption of isotropic velocity distribution yields the following Doppler broadening of the reaction rates operator:

$$
v \sigma_{T}(v)=\frac{1}{2} \int_{-1}^{1} d \mu \int_{v_{t} \mid v_{r}>0} v_{r} \sigma_{T_{0}}\left(v_{r}\right) \mathcal{M}_{T}\left(v_{t}\right) d v_{t}
$$


where $\mu=\overrightarrow{v_{t}} \cdot \vec{v}$ and $\mathcal{M}_{T}\left(v_{t}\right)$ is the Maxwellian distribution of speeds $v_{t}$ of the target particle at temperature $T$ :

$$
\mathcal{M}_{T}\left(v_{t}\right) d v_{t}=4 \pi v_{t}^{2}\left(s^{2} \frac{m_{n}}{2 \pi}\right)^{3 / 2} \mathrm{e}^{-s^{2}\left(\frac{1}{2} m_{n} v_{t}^{2}\right)} d v_{t}
$$

$m_{n}$ designates the neutron mass, and $s=\sqrt{\frac{A}{k_{b}\left(T-T_{0}\right)}}$, where $A$ is the atomic mass ratio of the target nuclei with respect to the incoming neutron, and $k_{b}$ is the Boltzmann constant.

Further developing the classical mechanics energy transformation

$$
\frac{1}{2} m v_{r}^{2}=\frac{1}{2} m\left(v^{2}-2 \mu v_{t} v+v_{t}^{2}\right)
$$

yields Solbrig's kernel for Doppler broadening as a function of $z=\sqrt{E}$, where $E=\frac{1}{2} m_{n} v^{2}$ is the kinetic energy of the incoming neutron [3]:

$$
\begin{aligned}
z^{2} \sigma_{T}(z) & =\int_{0}^{\infty} x^{2} \sigma_{T_{0}}(x) \mathcal{K}^{\mathbb{D}}(z, x) d x \\
\text { where: } \mathcal{K}^{\mathbb{D}}(z, x) & =\frac{s}{\sqrt{\pi}}\left[e^{-s^{2}(z-x)^{2}}-e^{-s^{2}(z+x)^{2}}\right]
\end{aligned}
$$

\subsection{Kernel operators definitions}

From the latter, it stems that Doppler broadening is a kernel linear operation that transforms a quantity at temperature $T_{0}$ to its value at temperature $T$. Depending on which of the following three quantities is being transformed, four kernels $\mathcal{K}$ can be observed:

1. The $E \cdot \sigma(E)$ quantity relates to the $\mathcal{K}_{T}^{\mathbb{B}}$ kernel - here called the Boltzmann kernel since it is the Boltzmann distribution of the target particles energies:

$$
z^{2} \sigma_{T}(z)=\int x^{2} \sigma_{T_{0}}(x) \mathcal{K}_{T}^{\mathbb{B}}(z, x) d x
$$

2. The $v \cdot \sigma(v)$ quantity can relate to two kernels, according to how the integration is interpreted: $\mathcal{K}_{T}^{\mathbb{M}}-$ here called the Maxwell kernel as it is the Maxwell velocity distribution of the target particles; or $\mathcal{K}_{T}^{\frac{\mathrm{M}}{v}}$ - here called the Maxwell-over-velocity kernel since that is the ratio that springs from the integration.

$$
\begin{aligned}
& v \sigma_{T}(v)=\int x \sigma_{T_{0}}(x) \mathcal{K}_{T}^{\mathbb{M}}(v, x) d x \\
& v \sigma_{T}(v)=\int x \sigma_{T_{0}}(x) \mathcal{K}_{T}^{\frac{\mathbb{M}}{v}}(v, x) d x
\end{aligned}
$$

3. The $\sigma(z)$ quantity relates to the kernel $\mathcal{K}_{T}^{\mathbb{D}}$ - here called the Doppler kernel as it is the kernel of the Doppler broadening operation acting on the cross section:

$$
\sigma_{T}(z)=\int \sigma_{T_{0}}(x) \mathcal{K}_{T}^{\mathbb{D}}(z, x) d x
$$

For clarity purposes, the integrals in the latter definitions are so-far willfully left vague, and formal derivations are expressed to present the structure of the reasoning. The details of all derivations are relegated to the appendixes for reproducibility purposes. 


\subsection{Kernel reconstruction}

Hereafter is presented the general method of kernel reconstruction, and the kernel reconstruction problem is formally solved in the general case for both free and constrained reconstruction problems.

\subsubsection{Temperature interpolation by linear combination of pre-tabulated cross sections}

Let us consider the cross section temperature interpolation problem and search for solutions that are linear combinations of the pre-tabulated values, such that we approximate the exact value of the cross section, $\sigma_{T}^{(\text {exact })}$ by:

$$
\sigma_{T}^{(\text {exact })} \approx \sigma_{T}^{(\text {approx })}=\sum_{j=1}^{N} c_{j} \sigma_{T_{j}}
$$

where the $\sigma_{T_{j}}$ are assumed to be exactly known. Our goal is to formulate the problem so as to find the optimal choice of $\left(c_{j}\right)$ coefficients to perform this linear interpolation.

The linearity of the kernel operators entails that the difference between the exact and the interpolated quantity translates into an integral difference between kernels. In the case of the Doppler kernel, this means:

$$
\left(\sigma_{T}^{(\text {exact })}-\sigma_{T}^{(\text {approx })}\right)(z)=\int \sigma_{T_{0}}(x)\left[\mathcal{K}_{T}^{\mathbb{D}}-\sum_{j} c_{j} \mathcal{K}_{T_{j}}^{\mathbb{D}}\right](z, x) d x
$$

which leads to the weak formulation:

$$
\int\left(\sigma_{T}^{(\text {exact })}-\sigma_{T}^{(\text {approx })}\right)(z) d z=\iint \sigma_{T_{0}}(x)\left[\mathcal{K}_{T}^{\mathbb{D}}-\sum_{j} c_{j} \mathcal{K}_{T_{j}}^{\mathbb{D}}\right](z, x) d x d z
$$

\subsubsection{Free kernel reconstruction $\&$ algebraic solution}

Regardless of the metrics chosen, the minimization of the latter expression will be cross section dependent. However, in order to develop a cross section independent interpolation method, one can search for the $\left(c_{j}\right)$ coefficients that minimize the distance between the kernels, $\left\|\mathcal{K}_{T}^{\mathbb{D}}-\sum_{j} c_{j} \mathcal{K}_{T_{j}}^{\mathbb{D}}\right\|$. At this point, the choice of the norm can be arbitrary. However, choosing the $\mathrm{L}_{2}$ norm will not only provide with a mathematically convenient framework which yields algebraically closed formulas, but is moreover physically justified by the general shape of nuclear cross sections, which yields a frequency separation argument presented in appendix D. This entails the following $\mathrm{L}_{2}$ minimization problem:

Find the $\left(c_{j}\right)$ coefficients that minimize in the $\mathrm{L}_{2}$ sense the difference between the exact Doppler kernel at temperature $T$ and a linear combination of kernels at temperatures $\left(T_{j}\right)$

$$
c_{j}=\underset{c_{j}}{\arg \min }\left\|\mathcal{K}_{T}^{\mathbb{D}}-\sum_{j} c_{j} \mathcal{K}_{T_{j}}^{\mathbb{D}}\right\|_{\mathrm{L}_{2}}
$$

Solving this problem is here called $\mathrm{L}_{2}$ free Doppler kernel reconstruction, and the same approach on $v \cdot \sigma_{T}(v)$ and $E \cdot \sigma_{T}(E)$ yields analog minimization problems and free kernel reconstructions for the Maxwell, Maxwell-over-velocity and Boltzmann kernels.

An important characteristic of these kernel reconstruction schemes is that the choice of the $\mathrm{L}_{2}$ norm provides an Hilbert space structure in which the latter optimization problems become algebraically solvable. 
Indeed, let $\left(c_{j}\right)$ be the coefficients we are searching for, and $\langle f \mid g\rangle$ denote the scalar product acting upon our Hilbert functional space, then developing $\left\|\mathcal{K}_{T}-\sum_{j=1}^{N} c_{j} \mathcal{K}_{T_{j}}\right\|_{\mathrm{L}_{2}}^{2}=\left\langle\mathcal{K}_{T} \mid \mathcal{K}_{T}\right\rangle-2 \sum_{j \in \llbracket 1 ; N \rrbracket} c_{j}\left\langle\mathcal{K}_{T_{j}} \mid \mathcal{K}_{T}\right\rangle+$ $\sum_{j, k} c_{k} c_{j}\left\langle\mathcal{K}_{T_{j}} \mid \mathcal{K}_{T_{k}}\right\rangle$, and searching for the local minima $\forall j \in \llbracket 1 ; N \rrbracket \frac{\partial}{\partial c_{j}}\left\|\mathcal{K}_{T}-\sum_{j=1}^{N} c_{j} \mathcal{K}_{T_{j}}\right\|_{L_{2}}^{2}=0$, yields the following system:

$$
\forall j \in \llbracket 1 ; N \rrbracket, \quad \sum_{k \neq j} c_{k}\left\langle\mathcal{K}_{T_{j}} \mid \mathcal{K}_{T_{k}}\right\rangle+c_{j}\left\langle\mathcal{K}_{T_{j}} \mid \mathcal{K}_{T_{j}}\right\rangle=\left\langle\mathcal{K}_{T_{j}} \mid \mathcal{K}_{T}\right\rangle
$$

Thus, if our problem has an interior solution, it must satisfy the above system, which can be written in matrix form as follows:

$$
\mathbb{G} \cdot C=Y
$$

where $C$ is the array of coefficients we are searching for:

$$
C=\operatorname{vect}\left(\left\langle\mathcal{K}_{T_{i}} \mid \mathcal{K}_{T}\right\rangle\right)=\left[\begin{array}{lll}
c_{1} & , \ldots, & c_{N}
\end{array}\right]^{\top}
$$

$\mathbb{G}$ is the Gram matrix of the kernels:

$$
\mathbb{G}=\operatorname{mat}\left(\left\langle\mathcal{K}_{T_{i}} \mid \mathcal{K}_{T_{j}}\right\rangle\right)
$$

and $Y$ is the target vector:

$$
Y=\left[\begin{array}{lll}
\left\langle\mathcal{K}_{T_{1}} \mid \mathcal{K}_{T}\right\rangle & , \ldots, \quad\left\langle\mathcal{K}_{T_{N}} \mid \mathcal{K}_{T}\right\rangle
\end{array}\right]^{\top}
$$

\subsubsection{Constrained kernel reconstruction \& Algebraic solution}

Both Boltzmann and Maxwell kernels are the respective densities of the Boltzmann and Maxwell probability distribution functions, which means that their $\mathrm{L}_{1}$ norm is equal to one.

When performing the kernel reconstruction, it can be advantageous to conserve this physical property, which guarantees the volume of the kernel is unchanged. This implies making sure that the linear combination of Boltzmann or Maxwell distributions integrates to one so that it can still be considered a probability density function for the target nuclei, or, equivalently, imposing that the linear interpolation coefficients $c_{j}$ sum up to 1 . This leads us to consider the $L_{2}$ constrained kernel reconstruction problems:

Find the $\left(c_{j}\right)$ coefficients that minimize in the $\mathrm{L}_{2}$ sense the difference between the exact kernel at temperature $T$ and a linear combination of kernels at temperatures $\left(T_{j}\right)$

$$
c_{j}=\underset{c_{j}}{\arg \min }\left\|\mathcal{K}_{T}-\sum_{j} c_{j} \mathcal{K}_{T_{j}}\right\|_{\mathrm{L}_{2}}
$$

under the unity constraint of conserving probability densities:

$$
\sum_{j=1}^{N} c_{j}=1
$$

In the constrained kernel reconstruction case, injecting the condition $\sum_{j} c_{j}=1$ into $\left\|\mathcal{K}_{T}-\sum_{j=1}^{N} c_{j} \mathcal{K}_{T_{j}}\right\|_{L_{2}}$ yields the following system:

$$
\left\{\begin{aligned}
\sum_{k \neq j}^{N-1} c_{k}\left\langle\widetilde{\mathcal{K}_{T_{j}}} \mid \widetilde{\mathcal{K}_{T_{k}}}\right\rangle+c_{j}\left\langle\widetilde{\mathcal{K}_{T_{j}}} \mid \widetilde{\mathcal{K}_{T_{j}}}\right\rangle & =\left\langle\widetilde{\mathcal{K}_{T_{j}}} \mid \widetilde{\mathcal{K}_{T}}\right\rangle \\
\forall j \in \llbracket 1 ; N-1 \rrbracket, \widetilde{\mathcal{K}_{T_{j}}} & =\mathcal{K}_{T_{j}}-\mathcal{K}_{T_{N}} \\
\sum_{j=1}^{N} c_{j} & =1
\end{aligned}\right.
$$


by noticing that

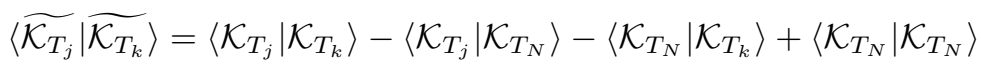

the constrained problem is thus reduced to inverting the following matrix system:

$$
\widetilde{\mathbb{G}} \cdot C=\widetilde{Y}
$$

where

and

$$
\left[\begin{array}{cccc}
\widetilde{g_{1,1}} & \cdots & \widetilde{g_{1, N-1}} & 0 \\
\vdots & \ddots & \vdots & \vdots \\
\widetilde{g_{N-1,1}} & \cdots & \widetilde{g_{N-1, N-1}} & 0 \\
1 & \cdots & 1 & 1
\end{array}\right]
$$

$$
\widetilde{Y}=\left[\widetilde{\left\langle\widetilde{\mathcal{K}_{T_{1}}} \mid \widetilde{\mathcal{K}_{T}}\right\rangle}, \ldots, \quad\left\langle\widetilde{\left\langle\overline{\mathcal{K}_{T_{N-1}}} \mid \widetilde{\mathcal{K}_{T}}\right\rangle, 1}\right]^{\top}\right.
$$

The actual expressions of these Gram matrices will now depend on the particular kernel being reconstructed and the scalar product considered.

\subsection{Dopper kernel reconstruction}

In the case of Doppler kernel reconstruction, the scalar products diverge. However, the algebraic solutions can still be derived with the help of a continuity argument exposed in appendix A. This yields the following temperature-dependent systems.

\subsubsection{Free Doppler reconstruction system}

The free Doppler kernel reconstruction system is:

$$
\mathbb{D} \cdot C=Y
$$

with

$$
\left[\begin{array}{ccc}
\frac{\sqrt{T_{1} T_{1}}}{\left(\frac{T_{1}+T_{1}}{2}\right)} & \cdots & \frac{\sqrt{T_{1} T_{N}}}{\left(\frac{T_{1}+T_{N}}{2}\right)} \\
\vdots & \ddots & \vdots \\
\frac{\sqrt{T_{1} T_{N}}}{\left(\frac{T_{1}+T_{N}}{2}\right)} & \cdots & \frac{\sqrt{T_{N} T_{N}}}{\left(\frac{T_{N}+T_{N}}{2}\right)}
\end{array}\right] \cdot\left[\begin{array}{c}
c_{1} \\
\vdots \\
c_{N}
\end{array}\right]=\left[\begin{array}{l}
\frac{\sqrt{T_{1} T}}{\left(\frac{T_{1}+T}{2}\right)} \\
\vdots \\
\frac{\sqrt{T_{N} T}}{\left(\frac{T_{N}+T}{2}\right)}
\end{array}\right]
$$

\subsubsection{Constrained Doppler reconstruction system}

The constrained Doppler kernel reconstruction system is:

$$
\widetilde{\mathbb{D}} \cdot C=\widetilde{A}
$$

where

$$
\left[\begin{array}{cccc}
\widetilde{d_{1,1}} & \cdots & \widetilde{d_{1, N-1}} & 0 \\
\vdots & \ddots & \vdots & \vdots \\
\widetilde{d_{N-1,1}} & \cdots & \widetilde{d_{N-1, N-1}} & 0 \\
1 & \cdots & 1 & 1
\end{array}\right] \cdot\left[\begin{array}{c}
c_{1} \\
\vdots \\
c_{N-1} \\
c_{N}
\end{array}\right]=\left[\begin{array}{l}
\widetilde{y_{1}} \\
\vdots \\
\widetilde{y_{N-1}} \\
1
\end{array}\right]
$$

with

$$
\widetilde{d_{i j}}=\frac{\sqrt{T_{i} T_{j}}}{\left(\frac{T_{i}+T_{j}}{2}\right)}-\frac{\sqrt{T_{i} T_{N}}}{\left(\frac{T_{i}+T_{N}}{2}\right)}-\frac{\sqrt{T_{N} T_{j}}}{\left(\frac{T_{N}+T_{j}}{2}\right)}+\frac{\sqrt{T_{N} T_{N}}}{\left(\frac{T_{N}+T_{N}}{2}\right)}
$$

and

$$
\widetilde{y_{i}}=\frac{\sqrt{T_{i} T}}{\left(\frac{T_{i}+T}{2}\right)}-\frac{\sqrt{T_{i} T_{N}}}{\left(\frac{T_{i}+T_{N}}{2}\right)}-\frac{\sqrt{T_{N} T}}{\left(\frac{T_{N}+T}{2}\right)}+\frac{\sqrt{T_{N} T_{N}}}{\left(\frac{T_{N}+T_{N}}{2}\right)}
$$




\subsubsection{Free Doppler reconstruction analytical solution}

Further algebraic manipulations, developed in appendix A, have also enabled to exhibit the analytical solution to the free Doppler kernel reconstruction system (i.e. equation (26)). The explicit formulae for the solution coefficients are:

$$
c_{j}=\frac{\sqrt{T_{j} T}}{\left(\frac{T_{j}+T}{2}\right)} \prod_{i \neq j}\left(\frac{T-T_{i}}{T+T_{i}}\right)\left(\frac{T_{j}+T_{i}}{T_{j}-T_{i}}\right)
$$

\subsection{Maxwell kernel reconstruction}

In the case of Maxwell kernel reconstruction, the algebraic solutions stem from the Gauss integrals. These are derived in appendix B, and yield the following temperature-dependent systems:

\subsubsection{Free Maxwell reconstruction system}

The free Mawell kernel reconstruction system is:

$$
\mathbb{M} \cdot C=Y
$$

with

$$
\left[\begin{array}{ccc}
\frac{T_{1} T_{1}}{\left(T_{1}+T_{1}\right)^{5 / 2}} & \cdots & \frac{T_{1} T_{N}}{\left(T_{1}+T_{N}\right)^{5 / 2}} \\
\vdots & \ddots & \vdots \\
\frac{T_{N} T_{1}}{\left(T_{N}+T_{1}\right)^{5 / 2}} & \cdots & \frac{T_{N} T_{N}}{\left(T_{N}+T_{N}\right)^{5 / 2}}
\end{array}\right] \cdot\left[\begin{array}{c}
c_{1} \\
\vdots \\
c_{N}
\end{array}\right]=\left[\begin{array}{l}
\frac{T_{1} T}{\left(T_{1}+T\right)^{5 / 2}} \\
\vdots \\
\frac{T_{N} T}{\left(T_{N}+T\right)^{5 / 2}}
\end{array}\right]
$$

\subsubsection{Constrained Maxwell reconstruction system}

The constrained Maxwell kernel reconstruction system is:

$$
\widetilde{\mathbb{M}} \cdot C=\widetilde{Y}
$$

where

$$
\left[\begin{array}{cccc}
\widetilde{m_{1,1}} & \ldots & \widetilde{m_{1, N-1}} & 0 \\
\vdots & \ddots & \vdots & \vdots \\
\widetilde{m_{N-1,1}} & \ldots & \widetilde{m_{N-1, N-1}} & 0 \\
1 & \cdots & 1 & 1
\end{array}\right] \cdot\left[\begin{array}{c}
c_{1} \\
\vdots \\
c_{N-1} \\
c_{N}
\end{array}\right]=\left[\begin{array}{l}
\widetilde{y_{1}} \\
\vdots \\
\widetilde{y_{N-1}} \\
1
\end{array}\right]
$$

with

$$
\widetilde{m_{i j}}=\frac{T_{i} T_{j}}{\left(T_{i}+T_{j}\right)^{5 / 2}}-\frac{T_{i} T_{N}}{\left(T_{i}+T_{N}\right)^{5 / 2}}-\frac{T_{N} T_{j}}{\left(T_{N}+T_{j}\right)^{5 / 2}}+\frac{T_{N} T_{N}}{\left(T_{N}+T_{N}\right)^{5 / 2}}
$$

and

$$
\widetilde{y_{i}}=\frac{T_{i} T}{\left(T_{i}+T\right)^{5 / 2}}-\frac{T_{i} T_{N}}{\left(T_{i}+T_{N}\right)^{5 / 2}}-\frac{T_{N} T}{\left(T_{N}+T\right)^{5 / 2}}+\frac{T_{N} T_{N}}{\left(T_{N}+T_{N}\right)^{5 / 2}}
$$

\subsection{Maxwell-over-velocity kernel reconstruction}

In the case of Maxwell-over-velocity kernel reconstruction, the algebraic solutions also stem from the Gauss integrals, but are of a different order due to a different interpretation of the integral domains. Derivations are found in appendix B, and yield the following temperature-dependent systems: 
2.6.1. Free Maxwell-over-velocity reconstruction system

The free Maxwell-over-velocity kernel reconstruction system is:

$$
\frac{\mathbb{M}}{v} \cdot C=Y
$$

with

$$
\left[\begin{array}{ccc}
\frac{1}{\sqrt{T_{1}+T_{1}}} & \cdots & \frac{1}{\sqrt{T_{1}+T_{N}}}{ }^{3} \\
\vdots & \ddots & \vdots \\
\frac{1}{{\sqrt{T_{N}+T_{1}}}^{3}} & \cdots & \frac{1}{\sqrt{T_{N}+T_{N}}}{ }^{3}
\end{array}\right] \cdot\left[\begin{array}{c}
c_{1} \\
\vdots \\
c_{N}
\end{array}\right]=\left[\begin{array}{l}
\frac{1}{\sqrt{T+T_{1}}}{ }^{3} \\
\vdots \\
\frac{1}{\sqrt{T+T_{N}}}{ }^{3}
\end{array}\right]
$$

It should be noted that this system is the Hadamard product $3^{\text {rd }}$ power of the Boltzmann kernel reconstruction problem that will be described below. This has the implication that the condition number of the system is essentially risen to the $3^{\text {rd }}$ power, significantly hindering the numerical stability of this system over the Boltzmann reconstruction one.

\subsubsection{Constrained Maxwell-over-velocity reconstruction system}

The constrained Maxwell-over-velocity kernel reconstruction system is:

$$
\frac{\widetilde{\mathbb{M}}}{\boldsymbol{v}} \cdot C=\widetilde{Y}
$$

where

$$
\left[\begin{array}{cccc}
\widetilde{m v_{1,1}} & \ldots & \widehat{m v_{1, N-1}} & 0 \\
\vdots & \ddots & \vdots & \vdots \\
\widetilde{m v_{N-1,1}} & \ldots & \widehat{m v_{N-1, N-1}} & 0 \\
1 & \cdots & 1 & 1
\end{array}\right] \cdot\left[\begin{array}{c}
c_{1} \\
\vdots \\
c_{N-1} \\
c_{N}
\end{array}\right]=\left[\begin{array}{l}
\widetilde{y_{1}} \\
\vdots \\
\widetilde{y_{N-1}} \\
1
\end{array}\right]
$$

with

$$
\widetilde{m v_{i j}}=\frac{1}{{\sqrt{T_{i}+T_{j}}}^{3}}-\frac{1}{{\sqrt{T_{i}+T_{N}}}^{3}}-\frac{1}{{\sqrt{T_{N}+T_{j}}}^{3}}+\frac{1}{{\sqrt{T_{N}+T_{N}}}^{3}}
$$

and

$$
\widetilde{y_{i}}=\frac{1}{{\sqrt{T_{i}+T^{3}}}^{3}}-\frac{1}{{\sqrt{T_{i}+T_{N}}}^{3}}-\frac{1}{{\sqrt{T_{N}+T^{3}}}^{3}}+\frac{1}{{\sqrt{T_{N}+T_{N}}}^{3}}
$$

\subsection{Boltzmann kernel reconstruction}

Boltzmann kernel reconstruction is rooted into Ferran's convolution formalization of the Doppler broadening operation [4]. Its Fourier analysis can help to physically justify the arbitrary choice of $\mathrm{L}_{2}$ norm reconstruction using a frequency separation argument, as reported in appendix D. The algebraic solutions for the Boltzmann kernel reconstruction are derived in appendix C, and yield the following temperaturedependent systems:

\subsubsection{Free Boltzmann reconstruction system}

The free Boltzmann kernel reconstruction system is:

$$
\mathbb{B} \cdot C=Y
$$

with

$$
\left[\begin{array}{ccc}
\frac{1}{\sqrt{T_{1}+T_{1}}} & \cdots & \frac{1}{\sqrt{T_{1}+T_{N}}} \\
\vdots & \ddots & \vdots \\
\frac{1}{\sqrt{T_{N}+T_{1}}} & \cdots & \frac{1}{\sqrt{T_{N}+T_{N}}}
\end{array}\right] \cdot\left[\begin{array}{c}
c_{1} \\
\vdots \\
c_{N}
\end{array}\right]=\left[\begin{array}{l}
\frac{1}{\sqrt{T+T_{1}}} \\
\vdots \\
\frac{1}{\sqrt{T+T_{N}}}
\end{array}\right]
$$




\subsubsection{Constrained Boltzmann reconstruction system}

The constrained Boltzmann kernel reconstruction system is:

$$
\widetilde{\mathbb{B}} \cdot C=\widetilde{Y}
$$

where

$$
\left[\begin{array}{cccc}
\widetilde{b_{1,1}} & \ldots & \widetilde{b_{1, N-1}} & 0 \\
\vdots & \ddots & \vdots & \vdots \\
\widetilde{b_{N-1,1}} & \ldots & \widehat{b_{N-1, N-1}} & 0 \\
1 & \cdots & 1 & 1
\end{array}\right] \cdot\left[\begin{array}{c}
c_{1} \\
\vdots \\
c_{N-1} \\
c_{N}
\end{array}\right]=\left[\begin{array}{l}
\widetilde{y_{1}} \\
\vdots \\
\widetilde{y_{N-1}} \\
1
\end{array}\right]
$$

with

$$
\widetilde{b_{i j}}=\frac{1}{\sqrt{T_{i}+T_{j}}}-\frac{1}{\sqrt{T_{i}+T_{N}}}-\frac{1}{\sqrt{T_{N}+T_{j}}}+\frac{1}{\sqrt{T_{N}+T_{N}}}
$$

and

$$
\widetilde{y_{i}}=\frac{1}{\sqrt{T_{i}+T}}-\frac{1}{\sqrt{T_{i}+T_{N}}}-\frac{1}{\sqrt{T_{N}+T}}+\frac{1}{\sqrt{T_{N}+T_{N}}}
$$

\section{Temperature-Optimized Kernel Reconstruction Doppler Broadening}

It has so far been demonstrated that provided the reference temperatures $\left(T_{j}\right)$ and a temperature of interest $T$ to which one wishes to interpolate the value of a Doppler broadened quantity by linear combination of the values at reference temperatures, then the choice of the $\left(c_{j}\right)$ interpolation coefficients can be optimally approximated in a cross section independent way by solving a kernel reconstruction problem. In other words, given $\left(T_{j}\right)$ and $T$, optimal choices (in $\mathrm{L}_{2}$ sense) for $\left(c_{j}\right)$ have been established.

The purpose of this section is to address the optimal choice of reference temperatures $\left(T_{j}\right)$ that will minimize the interpolation error over a given temperature range $T \in\left[T_{\min }, T_{\max }\right]$. This error is dependent on the cross section values. However, appendix D justifies why it is a good approximation to solve the interpolation problem in a cross section independent fashion by minimizing the distance amongst the kernels. From this it stems that the optimal choice of reference temperatures $\left(T_{j}\right)_{j \in \llbracket 1 ; N \rrbracket}$ can also be chosen in a cross section independent way by solving for the optimal set of reference temperatures $\left(T_{j}\right)_{j \in \llbracket 1 ; N \rrbracket}$ that minimizes the distance, in both energy and temperature spaces, and in a sense yet to be determined, amongst the kernels.

\section{1. $\mathrm{L}_{2}$ kernel reconstruction relative error}

Another advantage of having defined our problem with a Hilbert space structure is that the relative distances between the kernels also become analytically expressible as functions of the Gram matrix coefficients as follows:

$$
\left(\frac{\Delta \epsilon}{\epsilon}\right)_{\mathbb{G}} \equiv \frac{\left\|\mathcal{K}_{T}-\sum_{j=1}^{N} c_{j} \mathcal{K}_{T_{j}}\right\|_{L_{2}}}{\left\|\mathcal{K}_{T}\right\|_{L_{2}}}=\sqrt{1-2 \sum_{j} c_{j} \frac{\left\langle\mathcal{K}_{T_{i}} \mid \mathcal{K}_{T}\right\rangle}{\left\langle\mathcal{K}_{T} \mid \mathcal{K}_{T}\right\rangle}+\sum_{i} \sum_{j} c_{i} c_{j} \frac{\left\langle\mathcal{K}_{T_{i}} \mid \mathcal{K}_{T_{j}}\right\rangle}{\left\langle\mathcal{K}_{T} \mid \mathcal{K}_{T}\right\rangle}}
$$

Thus, for the various kernel reconstruction problems, one finds the following relative $\mathrm{L}_{2}$ distances among kernels by reporting the $\left(c_{j}\right)$, which are the solutions of either the constrained or free problem, into:

Boltzmann kernel relative error

$$
\left(\frac{\Delta \epsilon}{\epsilon}\right)_{\mathbb{B}} \equiv \frac{\left\|\mathcal{K}_{T}^{\mathbb{B}}-\sum_{j=1}^{N} c_{j} \mathcal{K}_{T_{j}}^{\mathbb{B}}\right\|_{L_{2}}}{\left\|\mathcal{K}_{T}^{\mathbb{B}}\right\|_{L_{2}}}=\sqrt{1-2 \sum_{j} c_{j} \frac{\sqrt{2 T}}{\sqrt{T_{j}+T}}+\sum_{i} \sum_{j} c_{i} c_{j} \frac{\sqrt{2 T}}{\sqrt{T_{i}+T_{j}}}}
$$


Maxwell kernel relative error

$$
\left(\frac{\Delta \epsilon}{\epsilon}\right)_{\mathbb{M}} \equiv \frac{\left\|\mathcal{K}_{T}^{\mathbb{M}}-\sum_{j=1}^{N} c_{j} \mathcal{K}_{T_{j}}^{\mathbb{M}}\right\|_{L_{2}}}{\left\|\mathcal{K}_{T}^{\mathbb{M}}\right\|_{L_{2}}}=\sqrt{1-2 \sum_{j} c_{j} \frac{T_{j} 2^{5 / 2} T^{3 / 2}}{\left(T_{j}+T\right)^{5 / 2}}+\sum_{i} \sum_{j} c_{i} c_{j} \frac{T_{i} T_{j} 2^{5 / 2} T^{1 / 2}}{\left(T_{i}+T_{j}\right)^{5 / 2}}}
$$

Maxwell-over-velocity kernel relative error

$$
\left(\frac{\Delta \epsilon}{\epsilon}\right)_{\frac{\mathbb{M}}{v}} \equiv \frac{\left\|\mathcal{K}_{T}^{\frac{\mathbb{M}}{v}}-\sum_{j=1}^{N} c_{j} \mathcal{K}_{T_{j}}^{\frac{\mathbb{M}}{v}}\right\|_{L_{2}}}{\left\|\mathcal{K}_{T}^{\frac{M}{v}}\right\|_{L_{2}}}=\sqrt{1-2 \sum_{j} c_{j} \frac{{\sqrt{2 T^{3}}}^{3}}{{\sqrt{T_{j}+T^{3}}}^{3}}+\sum_{i} \sum_{j} c_{i} c_{j} \frac{{\sqrt{2 T^{3}}}^{3}}{{\sqrt{T_{i}+T_{j}}}^{3}}}
$$

Doppler kernel relative error

$$
\left(\frac{\Delta \epsilon}{\epsilon}\right)_{\mathbb{D}} \equiv \frac{\left\|\mathcal{K}_{T}^{\mathbb{D}}-\sum_{j=1}^{N} c_{j} \mathcal{K}_{T_{j}}^{\mathbb{D}}\right\|_{\mathrm{L}_{2}}}{\left\|\mathcal{K}_{T}^{\mathbb{D}}\right\|_{\mathrm{L}_{2}}}=\sqrt{1-2 \sum_{i} c_{i} \frac{\sqrt{T_{i} T}}{\left(\frac{T_{i}+T}{2}\right)}+\sum_{i} \sum_{j} c_{i} c_{j} \frac{\sqrt{T_{i} T_{j}}}{\left(\frac{T_{i}+T_{j}}{2}\right)}}
$$

\section{2. $\mathrm{L}_{\infty}$ Temperature Optimization: Min-max problem}

These kernel reconstruction errors can now be optimized so as to find the reference temperatures $\left(T_{j}\right)$ that best reconstruct the kernel over a given temperature range. If the $\mathrm{L}_{\infty}$ metric is now chosen to represent what it means to minimize the distance among kernels over all of the temperature range, the optimization can be cast as solving for the following min-max problem:

Find the $\left(T_{j}\right)$ reference temperatures that minimize

the relative distance between exact and interpolated kernels

$$
\left(T_{j}\right)=\underset{\left(T_{j}\right)}{\arg \min }\left(\max _{T \in\left[T_{\min }, T_{\max }\right]}\left(\frac{\Delta \epsilon}{\epsilon}\right)_{\mathbb{G}}\right)
$$

over a range of temperatures of interest $T \in\left[T_{\min }, T_{\max }\right]$

\subsection{Solving the min-max problem: Temperature Optimization Algorithm}

For a given number $N$ of reference temperatures $\left(T_{j}\right)$, solving this min-max problem for any of the four kernels (and the respective eight solutions of optimal coefficients $\left(c_{j}\right)$ for free and constrained problems) is a highly non-linear (nor convex) high-dimensionality problem. Differential evolution and basin-hopping optimization algorithms were tested in an attempt to minimize the maximum relative error (i.e. $\left.\max \left(\frac{\Delta \epsilon}{\epsilon}\right)_{\mathbb{G}}\right)$ over the temperature range $\left[T_{\min }, T_{\max }\right]$. However, the high dimensionality of the optimization problem coupled with the stochastic nature of these algorithms led to the algorithms converging in many cases to local rather than global minimae, and with very long running times that were prohibitive past $N=6$. This issue inspired the creation of a non-stochastic optimization algorithm specifically tailored to the needs of solving the min-max problem on $\left(\frac{\Delta \epsilon}{\epsilon}\right)_{\mathbb{G}}$.

This algorithm works by choosing values for the reference temperatures such that the maximum relative error on the interval between any pair of adjacent reference temperatures is equal to within some tolerance to the maximum relative error between neighboring adjacent reference temperature pairs. In other words, given some number $N$ of reference temperatures, the algorithm chooses reference temperature values so that:

$$
\forall(i, j) \in \llbracket 1, N-1 \rrbracket^{2},\left(\max _{T \in\left[T_{i}, T_{i+1}\right]}\left(\frac{\Delta \epsilon}{\epsilon}\right)_{\mathbb{G}}\right) \approx\left(\max _{T \in\left[T_{j}, T_{j+1}\right]}\left(\frac{\Delta \epsilon}{\epsilon}\right)_{\mathbb{G}}\right)
$$

The algorithm begins with an initial linear guess in $\left(T_{j}\right)$, then iterates through sets of three adjacent reference temperatures, equalizing the maximum relative errors on the intervals between the first two and 
last two temperatures in each set. This is accomplished by moving the center temperature in a binary search fashion until the difference in the maximum errors on each of the two intervals is below some threshold.

The use of this algorithm allowed reference temperature placement to be optimized more accurately and in a much faster manner than either of the previously tried algorithms. The result of the algorithm on six reference temperatures with an initial guess of linear spacing is shown in figure (1). A thorough analysis of the optimal reference temperature grid for Doppler kernel reconstruction can be found in appendix E.

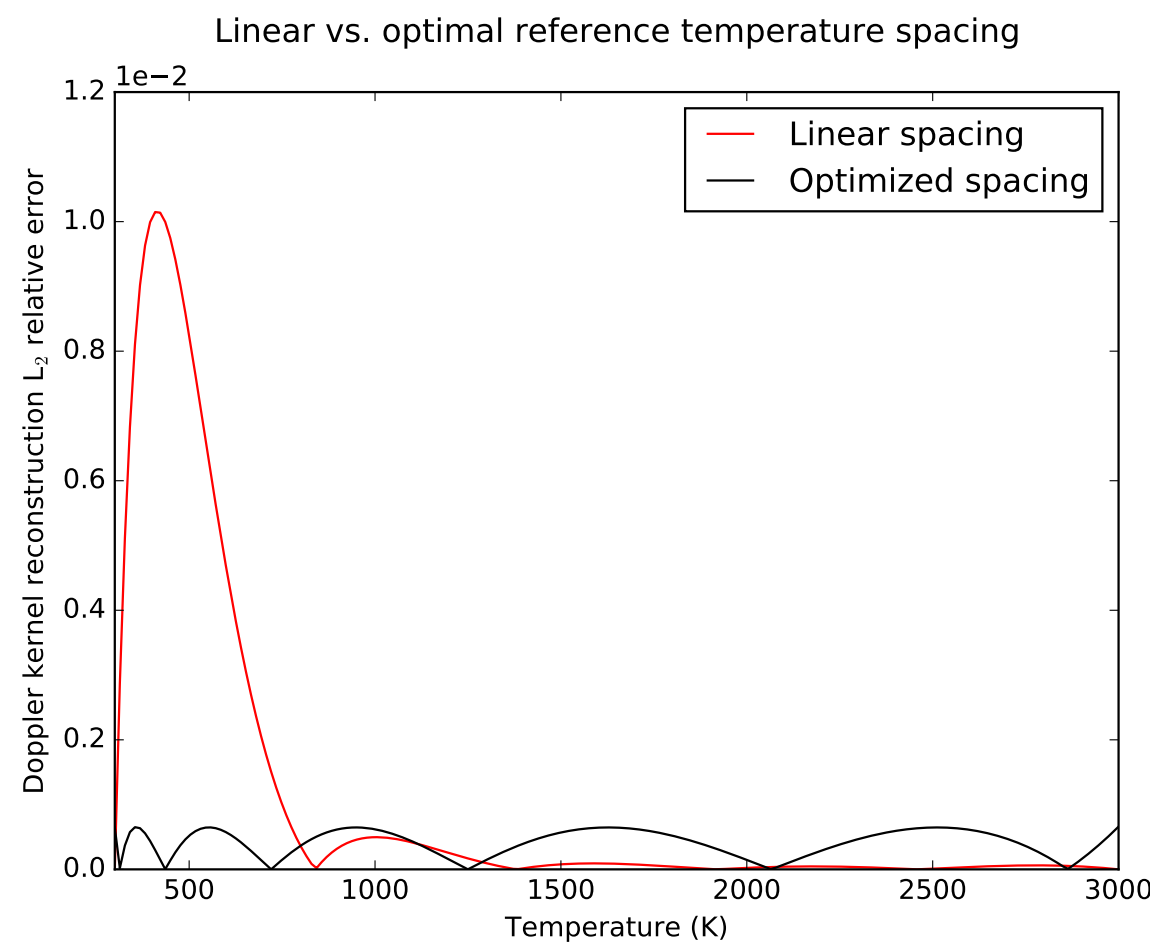

Figure 1: Free Doppler kernel reconstruction $L_{2}$ relative error over the temperature range [300, 3000] for both linearly spaced and optimized spacing of $N=6$ reference temperatures. Optimization of the reference temperature grid was performed through the presented algorithm with tolerance $10^{-6}$, and yields an order of magnitude on kernel reconstruction $\mathrm{L}_{2}$ relative error.

The high condition numbers of the matrices, specially the one stemming from equation (39), necessitated the use of the preconditioned conjugate gradients method for numerically stable inversion. For the free Doppler Kernel method in particular, numerical instability in the calculation of $\left(c_{j}\right)$ values was essentially eliminated through use of the analytical expression for these values shown in equation (31).

Another source of numerical instability was the truncation of calculated relative errors due to machine precision when the number of reference temperatures used was sufficiently large. This issue was addressed by calculating both relative errors and optimal temperature points in arbitrary precision. For any given $N$ number of reference temperatures, $10^{-(N+4)}$ precision was used to compute $\left(\frac{\Delta \epsilon}{\epsilon}\right)_{\mathbb{G}}^{2}$.

\subsection{Optimal reference $\left(T_{j}\right)$ distributions kernel reconstruction performance}

Once the optimal temperature grid $\left(T_{j}\right)$ has been found for each one of the eight methods, a way of gauging the performance of the various kernel reconstruction methods is to compare, given $N$ reference temperatures, which method is better at reconstructing its own kernel. The results of such an analysis are pictured in figure (2).

It can be observed that the temperature-optimized free Boltzmann kernel reconstruction was the best at reconstructing itself. This, however, did not mean it would fair better on actual isotopes. 


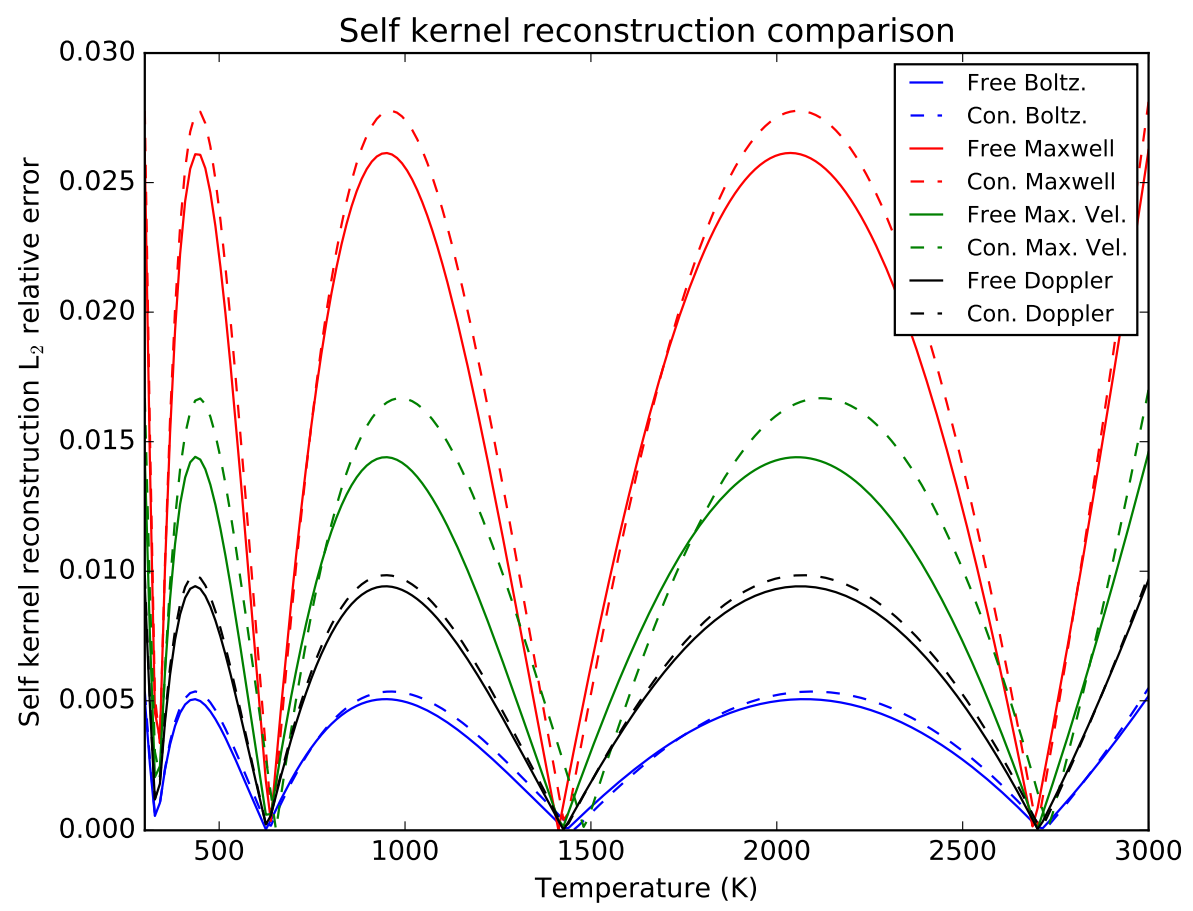

Figure 2: Comparison of self reconstruction ability: $\left(\frac{\Delta \epsilon}{\epsilon}\right)_{\mathbb{G}}(T)$ for each of the eight kernel reconstruction methods (free and constrained Boltzmann, Maxwell, Maxwell-over-velocity, and Doppler), with there respective optimal reference temperature grids $\left(T_{j}\right)$ for $N=4$ optimal reference temperatures over the temperature range [300, 3000]. The temperature-optimized free Boltzmann kernel reconstruction is the best at reconstructing itself.

\section{Performance \& comparison of temperature interpolation methods}

Of the eight Doppler broadening temperature interpolation methods here introduced, only one - the constrained Maxwell kernel reconstruction method - has been previously attempted [2]. The other linear interpolation methods traditionally used in the field are provided below, as well as the reference logarithmic interpolation method for comparison purposes [1].

\subsection{Previous temperature interpolation methods}

Traditional temperature interpolation methods have been either linear interpolations in temperature, or linear in logarithmic space, while a recent curve-fit method was introduced in MCNP.

\subsubsection{Linear interpolation methods}

Linear combination interpolation methods are defined by:

$$
\sigma_{T} \approx \sum_{j=1}^{N} c_{j} \sigma_{T_{j}}
$$

The choices for finding the $\left(c_{j}\right)$ have been [2]:

- The "Lin-Lin" interpolation scheme:

$$
c_{j}=\frac{\prod_{i \neq j}\left(T-T_{i}\right)}{\prod_{i \neq j}\left(T_{j}-T_{i}\right)}
$$


- The "Lin-Sqrt" interpolation scheme:

$$
c_{j}=\frac{\prod_{i \neq j}\left(\sqrt{T}-\sqrt{T_{i}}\right)}{\prod_{i \neq j}\left(\sqrt{T_{j}}-\sqrt{T_{i}}\right)}
$$

- The "Lin-Log" interpolation scheme:

$$
c_{j}=\frac{\prod_{i \neq j}\left(\ln T-\ln T_{i}\right)}{\prod_{i \neq j}\left(\ln T_{j}-\ln T_{i}\right)}
$$

\subsubsection{Logarithmic-Logarithmic method}

The "Log-Log" interpolation scheme is defined as:

$$
\begin{aligned}
\sigma_{T} & \approx \prod_{j=1}^{N}\left(\sigma_{T_{j}}\right)^{c_{j}} \\
c_{j} & =\frac{\prod_{i \neq j}\left(\ln T-\ln T_{i}\right)}{\prod_{i \neq j}\left(\ln T_{j}-\ln T_{i}\right)}
\end{aligned}
$$

Though they are here defined with general expressions, these interpolation methods as such would suffer from Runge phenomena, and are thus in practice always computed piecewise, using the two closest reference temperature points and ignoring the information provided by all other temperatures. The following comparisons use this two-points interpolation definition.

\subsubsection{MCNP curve fit method}

One other way to perform temperature interpolation of nuclear data is to curve fit the temperature dimension. This is the method recently adopted by MCNP [5]. For a given reaction, the cross section is Doppler broadened to a large number of temperatures on an union energy mesh. Then, for each energy, the coefficients, $a_{g, i}, b_{g, i}$ and $c_{g}$ are calculated for the following equation using least-squares minimization:

$$
\sigma_{\gamma}\left(T, E_{g}\right) \approx \sum_{i=1}^{N} \frac{a_{g, i}}{T^{i / 2}}+\sum_{i=1}^{N} b_{g, i} T^{i / 2}+c_{g}
$$

The MCNP method is thus a symmetrically truncated Laurent development curve-fit, and the coefficients are entirely cross section and energy dependent.

\subsection{Performance of the temperature-optimized kernel reconstruction methods}

The performance of an interpolation method is assessed on the $\mathrm{L}_{\infty}$ maximum relative error criterium as defined below:.

$$
\left|\frac{\Delta \sigma}{\sigma}\right|_{\mathrm{L}_{\infty}}(T)=\max _{E \in\left[E_{\min }, E_{\max }\right]}\left|\frac{\sigma_{T}^{(\text {exact })}(E)-\sigma_{T}^{(\text {approx })}(E)}{\sigma_{T}^{(\text {exact })}(E)}\right|
$$

and

$$
\left\|\frac{\Delta \sigma}{\sigma}\right\|_{\mathrm{L}_{\infty}}=\max _{T \in\left[T_{\min }, T_{\max }\right]}\left\{\left|\frac{\Delta \sigma}{\sigma}\right|_{\mathrm{L}_{\infty}}(T)\right\}
$$

The performance of the kernel reconstruction methods here introduced was tested upon isotope ${ }^{238} \mathrm{U}$, over the energy range $\left[E_{\min }=10^{-5} \mathrm{eV}, E_{\max }=20 \mathrm{keV}\right]$. The point-wise ENDF/B-VII.1 data was Doppler broadened using the SIGMA1 algorithm [3], to a sequence of fixed reference temperatures $\left(T_{j}\right)$, providing the exact reference $\sigma_{T_{j}}(E)$, as well as to calculate all $\sigma_{T}^{(\text {exact })}(E)$ of the temperature grid. The $\mathrm{L}_{\infty}$ norm of the relative difference between the exact cross section, $\sigma_{T}^{(\text {exact })}(E)$, and the interpolated cross section, $\sigma_{T}^{(\text {approx })}(E)$, was then computed for a range of temperatures. 


\subsubsection{Best performer amongst kernel reconstruction methods}

Each of the eight temperature-optimized kernel reconstruction methods was run using one to six reference temperatures on ${ }^{238} \mathrm{U}$ isotope data. The result of this test for $N=6$ is displayed in figure (3). It can be noticed that the constrained interpolations slightly outperform the free ones in this case. This could presumably be attributed to the fact that constraining the coefficients to summing to unity enforced the essential property of probability distribution, yielding better results overall, specially at the tails of the distributions. Thus, though the free $\frac{\mathbb{M}}{v}$ method does not appear in figure (3) due to numerical instability issues, the constrained version of this method can be viewed as its lower bound.

From figure (3), it can readily be observed that the two Doppler reconstruction methods outperform all other methods over the entire range of temperatures. Though the temperature-optimized constrained Doppler kernel reconstruction method narrowly outperforms the temperature-optimized free Doppler kernel reconstruction one, the difference in performance is quite slight. The ease with which the free Doppler kernel reconstruction coefficients can be computed using the analytical expression of equation (31), as opposed to the matrix inversion required to compute the constrained Doppler coefficients, was felt to make up for the small performance difference between the two methods. Thus the temperature-optimized free Doppler kernel reconstruction method was reckoned to be the best performer amongst temperature-optimized kernel reconstruction methods, and was thus tested on ${ }^{238} \mathrm{U}$ isotope and compared against currently used Doppler broadening interpolation methods.

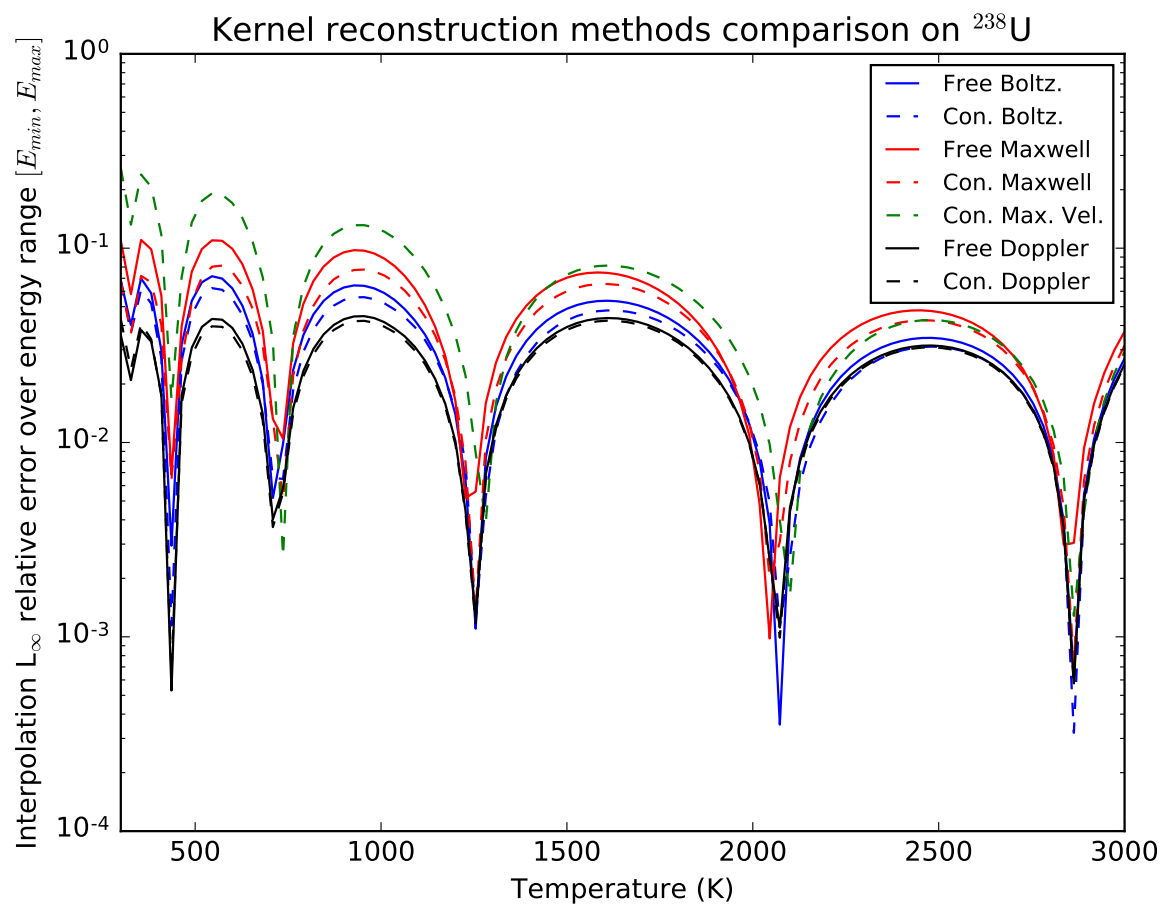

Figure 3: $\left|\frac{\Delta \sigma}{\sigma}\right|_{\mathrm{L}_{\infty}}(T)$ is computed for $N=6$ optimal reference temperatures on the temperature range [300, 3000], for each kernel reconstruction method. The two Doppler kernel reconstruction methods outperform all other kernel reconstruction methods on ${ }^{238} \mathrm{U}$ isotope. The ease of computing the free Doppler kernel reconstruction coefficients led to its choice over the constrained Doppler method as a candidate for further testing.

\subsubsection{Performance of temperature-optimized free Doppler kernel reconstruction against previous methods}

The temperature-optimized free Doppler kernel reconstruction method was tested on ${ }^{238} \mathrm{U}$ isotope data against linear interpolation, logarithmic interpolation, and the MCNP curve fit method discussed in section 
4.1.3 for $N=2$ through 15 reference temperatures. The results of this are displayed in figure (4). The free Doppler kernel reconstruction method was found to beat the other methods up to $N=12$, at which point the maximum relative errors of the kernel reconstruction and the MCNP curve fit methods fell below the level of accuracy from the SIGMA1 Doppler broadening of the ${ }^{238} \mathrm{U}$ isotope data itself, rendering comparison meaningless. From figure (4), it can be observed that in order to obtain $0.1 \%$ accuracy on a reconstructed ${ }^{238} \mathrm{U}$ kernel using the temperature-optimized free Doppler kernel reconstruction method, nine reference temperatures would be required. The ${ }^{238} \mathrm{U}$ isotope was chosen because the complexity of its resonance structure makes it a difficult isotope to Doppler broaden. Therefore it is likely that using the free Doppler kernel reconstruction method with $N=10$ reference temperatures would be sufficient to ensure $0.1 \%$ precision on all other isotopes.

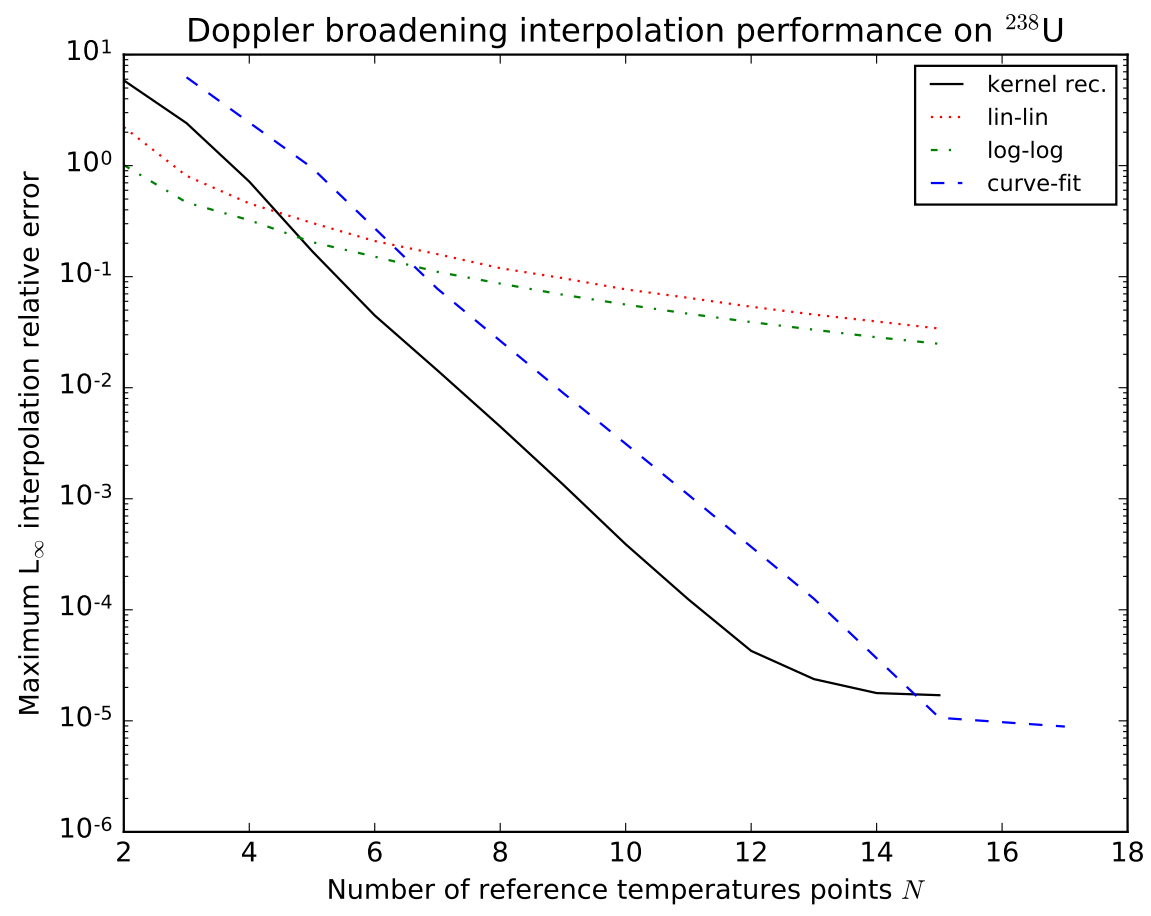

Figure 4: Maximum relative errors over energy $\left[10^{-5} \mathrm{eV}, 20 \mathrm{keV}\right]$ and temperature $[300 \mathrm{~K}, 3000 \mathrm{~K}]$ ranges. Performance of temperature-optimized free Doppler kernel reconstruction method (kernel rec.) compared to linear interpolation (lin-lin), logarithmic interpolation (log-log), and the MCNP curve fit (curve-fit) method. Temperatureoptimized free Doppler kernel reconstruction is one order of magnitude more accurate than the MCNP curve-fit method. $N=10$ optimal reference temperatures suffice to achieve $0.1 \%$ relative error.

\subsubsection{Considerations on performance}

Apart from the MCNP curve fit method, which is an ad hoc Laurent development curve-fit which kernel reconstruction outperforms by one order of magnitude on precision of interpolation for the same number of reference temperatures, previous methods all consisted of a local interpolation between the two closest temperatures. For a small-enough temperature difference $\left(T_{j+1}-T_{j} \rightarrow 0\right)$, lin-lin interpolation is sure to converge as a $1^{\text {st }}$ order Taylor expansion, with convergence in $\mathcal{O}(N)$. In contrast, the kernel reconstruction methods here introduced are global methods. They converge as $\mathcal{O}\left(C^{N}\right)$ to the true solution as the number of reference temperatures increases $(N \rightarrow \infty)$, because the physics of the system at temperature $T$ are increasingly well reconstructed through linear combinations of distributions at reference temperatures $T_{j}$.

Analyses have shown that to achieve $0.1 \%$ accuracy on temperature interpolation of ${ }^{238} \mathrm{U}$, previous 
methods require a temperature mesh of about $30 \mathrm{~K}$ intervals, thus requiring roughly 100 temperature points to cover nuclear reactor analysis range [1]. The need to use the cross section information for all 10 reference temperatures in the kernel reconstruction methods, instead of only the 2 closest for the older methods (except the MCNP curve fit which needs 12 points for the same precision), invariably requires more cross section lookups. However, the same accuracy can be achieved with roughly one order of magnitude less temperature points. Additionally, better efficiency can be obtained if each nuclide utilizes a uniform energy grid in temperature and proper ordering.

\section{Conclusions}

A new family of methods has here been established for performing cross section, reaction rates, or energy times cross section, temperature interpolation by linear combination of reference cross sections (or their respective quantity). The methods rely on the $\mathrm{L}_{2}$-norm difference minimization of operator kernels, representing the physical idea that the optimal way of interpolating is by best reconstructing the conditions of the interaction, that is the kernel of the operation. For instance, this can mean reconstructing the energy (or velocity) distribution for the target nuclei as close as possible to the real one at temperature $T$. Furthermore, isotope-independent optimal reference temperatures grids have been calculated to fine-tune the performance of kernel reconstruction methods over a given temperature range $\left[T_{\min }, T_{\max }\right]$. Though these methods require higher runtime due to multiple cross section lookups, the temperature-optimized free Doppler kernel reconstruction method was shown to achieve $0.1 \%$ accuracy on isotope ${ }^{238} \mathrm{U}$ cross section temperature interpolation over the entire temperature range $T \in[300 K, 3000 K]$ with only $N=10$ optimally spaced reference temperatures $\left(T_{j}\right)$, reducing the memory requirements for Doppler broadening interpolation by an order of magnitude.

\section{Acknowledgments}

The first author was supported by the Consortium for Advanced Simulation of Light Water Reactors (CASL), an Energy Innovation Hub for Modeling and Simulation of Nuclear Reactors under U.S. Department of Energy Contract No. DE-AC05-00OR22725. The second author was partially supported under U.S. Department of Energy Nuclear Energy University Programs Graduate Fellowship. The third author was partially supported by the Massachusetts Institute of Technology Undergraduate Research Opportunity Program.

\section{A. Doppler kernel reconstruction derivations}

\section{A.1. Dopper kernel reconstruction divergence issue}

The cross section is extended as an odd function over the entire energy range as:

$$
s_{T}: E \mapsto\left\{\begin{aligned}
\sigma_{T}(E), & \forall E \in \mathbb{R}_{+} \\
s_{T}(E)=-s_{T}(-E) & \forall E \in \mathbb{R}_{-}
\end{aligned}\right.
$$

which transforms Solbrig's kernel into the following Doppler broadening operation:

$$
s_{T}(z)=\int_{-\infty}^{\infty} s_{T_{0}}(x) \mathcal{K}_{T}^{\mathbb{D}}(z, x) d x
$$

with $z=\sqrt{E}$ and where the Doppler Kernel $\mathcal{K}_{T}^{\mathbb{D}}$ is here defined as

$$
\mathcal{K}_{T}^{\mathbb{D}}(z, x) \equiv \frac{x^{2}}{z^{2}} \mathcal{K}_{T}^{\mathbb{B}}(z-x)=\frac{x^{2}}{z^{2}} \frac{s}{\sqrt{\pi}} \mathrm{e}^{-s^{2}(z-x)^{2}}
$$

The functional Hilbert space is provided with the following scalar product 


$$
\langle f \mid g\rangle \equiv \iint_{\mathbb{R}^{2}} f \cdot g
$$

With this notation, and defining $\xi_{i}=\frac{1}{4 s_{i}^{2}} \propto T_{i}$, it can be shown that

$$
\left\langle\mathcal{K}_{T_{i}}^{\mathbb{D}} \mid \mathcal{K}_{T_{j}}^{\mathbb{D}}\right\rangle=\frac{1}{2 \pi} \frac{\sqrt{\xi_{i} \xi_{j}}}{\xi_{i}+\xi_{j}} \Theta
$$

where:

$$
\Theta=2 \int_{\theta=0}^{\pi} \frac{\cos ^{4} \theta}{\sin ^{4} \theta(\cos \theta-\sin \theta)^{2}} d \theta
$$

One can observe that $\Theta$ diverges: the integrand is defined everywhere but for the points: $\left\{\theta=0, \theta=\frac{\pi}{4}, \theta=\pi\right\}$. The problem thus seems to be ill-defined.

\section{A.1.1. Free Doppler kernel reconstruction}

It is however still possible to find an optimal solution to the problem by means of a continuity argument. Defining $\Theta_{\epsilon}$ as:

$$
\Theta_{\epsilon}=2 \int_{\epsilon}^{\frac{\pi}{4}-\epsilon} \frac{\cos ^{4} \theta}{\sin ^{4} \theta(\cos \theta-\sin \theta)^{2}} d \theta+2 \int_{\frac{\pi}{4}+\epsilon}^{\pi-\epsilon} \frac{\cos ^{4} \theta}{\sin ^{4} \theta(\cos \theta-\sin \theta)^{2}} d \theta
$$

For all value of epsilon $\epsilon>0$, the optimal inversion of the system becomes:

$$
\mathbb{D}_{\epsilon} \cdot C=Y_{\epsilon}
$$

with

$$
\left[\begin{array}{ccc}
\frac{\sqrt{T_{1} T_{1}}}{\left(\frac{T_{1}+T_{1}}{2}\right)} & \cdots & \frac{\sqrt{T_{1} T_{N}}}{\left(\frac{T_{1}+T_{N}}{2}\right)} \\
\vdots & \ddots & \vdots \\
\frac{\sqrt{T_{1} T_{N}}}{\left(\frac{T_{1}+T_{N}}{2}\right)} & \cdots & \frac{\sqrt{T_{N} T_{N}}}{\left(\frac{T_{N}+T_{N}}{2}\right)}
\end{array}\right] \cdot\left[\begin{array}{c}
c_{1} \\
\vdots \\
c_{N}
\end{array}\right]=\left[\begin{array}{c}
\frac{\sqrt{T_{1} T}}{\left(\frac{T_{1}+T}{2}\right)} \\
\vdots \\
\frac{\sqrt{T_{N} T}}{\left(\frac{T_{N}+T}{2}\right)}
\end{array}\right]
$$

where the definition of $\xi_{j}=\frac{k_{b}\left(T_{j}-T_{0}\right)}{4 A}$ was used to show the explicit temperature dependence of the Gram coefficients, and also making the problem nuclide independent. Since the system is invariant in $\epsilon$, by continuity of the property with respect to the value of $\epsilon$, this system still represents the optimal solution in the limit of $\epsilon=0$.

\section{A.1.2. Constrained Doppler kernel reconstruction}

As done above, the constrained problem becomes

$$
\widetilde{\mathbb{D}} \cdot C=\widetilde{A}
$$

where

$$
\left[\begin{array}{cccc}
\widetilde{d_{1,1}} & \cdots & \widetilde{d_{1, N-1}} & 0 \\
\vdots & \ddots & \vdots & \vdots \\
\widetilde{d_{N-1,1}} & \cdots & \widetilde{d_{N-1, N-1}} & 0 \\
1 & \cdots & 1 & 1
\end{array}\right] \cdot\left[\begin{array}{c}
c_{1} \\
\vdots \\
c_{N-1} \\
c_{N}
\end{array}\right]=\left[\begin{array}{l}
\widetilde{y_{1}} \\
\vdots \\
\widetilde{y_{N-1}} \\
1
\end{array}\right]
$$

with

$$
\widetilde{d_{i j}}=\frac{\sqrt{T_{i} T_{j}}}{\left(\frac{T_{i}+T_{j}}{2}\right)}-\frac{\sqrt{T_{i} T_{N}}}{\left(\frac{T_{i}+T_{N}}{2}\right)}-\frac{\sqrt{T_{N} T_{j}}}{\left(\frac{T_{N}+T_{j}}{2}\right)}+\frac{\sqrt{T_{N} T_{N}}}{\left(\frac{T_{N}+T_{N}}{2}\right)}
$$


and

$$
\widetilde{y_{i}}=\frac{\sqrt{T_{i} T}}{\left(\frac{T_{i}+T}{2}\right)}-\frac{\sqrt{T_{i} T_{N}}}{\left(\frac{T_{i}+T_{N}}{2}\right)}-\frac{\sqrt{T_{N} T}}{\left(\frac{T_{N}+T}{2}\right)}+\frac{\sqrt{T_{N} T_{N}}}{\left(\frac{T_{N}+T_{N}}{2}\right)}
$$

\section{A.2. Analytical Solutions}

The free Doppler kernel reconstruction system has stunningly remarkable properties:

1. Since $\mathbb{D}$ is a Gram matrix, it is a strictly symmetric positive definite matrix: $\mathbb{D} \in \mathcal{S}_{N}^{++}$

2. Moreover, the elements of the matrix $\mathbb{D}$ are ratios of the geometric mean to the arithmetic mean of the reference temperature pairs, which only yields values between 0 and 1, with 1's on the diagonal: $\frac{\sqrt{T_{i} T_{j}}}{\left(\frac{T_{i}+T_{j}}{2}\right)} \in[0,1]$. This quantity is very rich in physical meaning and has links to the information entropy of the system.

3. The matrix $\mathbb{D}$ also exhibits a particular symmetry in that it can be written as:

$$
\mathbb{D}=2 \cdot \mathbb{T}^{1 / 2} \cdot \mathbb{C} \cdot \mathbb{T}^{1 / 2}
$$

where $\mathbb{T}=\operatorname{diag}\left(T_{i}\right)$ and $\mathbb{C}=\operatorname{mat}\left(\frac{1}{T_{i}+T_{j}}\right)$. The latter is a Cauchy matrix, which enables us to derive the algebraic solution to this system.

Cramer's inversion formula on the free Doppler kernel reconstruction system yields:

$$
c_{k}=\frac{\operatorname{det}\left(\mathbb{D}_{(k)}\right)}{\operatorname{det}(\mathbb{D})}
$$

where $\mathbb{D}_{(k)}$ is the matrix formed by replacing the $k$-th column of $\mathbb{D}$ by the column vector $Y$.

Let us note that $\mathbb{D}_{(k)}$ also exhibits a particular symmetry in that it can be written:

$$
\mathbb{D}_{(k)}=2 \cdot \mathbb{T}_{(k)}^{1 / 2} \cdot \mathbb{C}_{(k)} \cdot \mathbb{T}^{1 / 2}
$$

where the sub-index $(k)$ signifies that $T_{k}$ is replaced by $T$ in the $k$-th column:

$$
\begin{aligned}
& \mathbb{T}_{(k)}=\operatorname{diag}\left(T_{1}, \ldots, T_{k-1}, T, T_{k+1}, \ldots, T_{N}\right) \text { and } \\
& \mathbb{C}_{(k)}=\operatorname{mat}\left(\mathbb{C}_{1}, \ldots, \mathbb{C}_{k-1},\left(\frac{1}{T_{i}+T}\right)_{i}, \mathbb{C}_{k+1}, \ldots, \mathbb{C}_{N}\right) .
\end{aligned}
$$

Both $\mathbb{C}$ and $\mathbb{C}_{(k)}$ are Cauchy matrices of the type mat $\left(\frac{1}{a_{i}+b_{j}}\right)$, the determinant of which is given by the well-known formula:

$$
|\mathbb{C}|_{N}=\frac{\prod_{i<j}\left(a_{j}-a_{i}\right) \prod_{i<j}\left(b_{j}-b_{i}\right)}{\prod_{i, j}\left(a_{i}+b_{j}\right)}
$$

By analyzing the ratio of determinants in Cramer's formula $c_{k}=\frac{\sqrt{T} \operatorname{det}\left(\mathbb{C}_{k}\right)}{\sqrt{T_{k}} \operatorname{det}(\mathbb{C})}$, one finds the explicit solution of the kernel-minimizing coefficients:

$$
c_{k}=\frac{\sqrt{T_{k} T}}{\left(\frac{T_{k}+T}{2}\right)} \prod_{i \neq k}\left(\frac{T-T_{i}}{T+T_{i}}\right)\left(\frac{T_{k}+T_{i}}{T_{k}-T_{i}}\right)
$$

It is noteworthy that $c_{k}(\sqrt{T})$ is a proper rational fraction in $\sqrt{T}$ of degree -1 , and on which it is thus possible to perform partial fraction decomposition, with poles $\pm i \sqrt{T_{i}}$ and roots $\pm \sqrt{T_{i}}$ and 0 .

Thus, provided a set of reference temperatures $\left(T_{j}\right)$ and given a temperature $T$, the $\left(c_{k}\right)$ coefficients in equation A.18 are the ones that algebraically minimize the $\mathrm{L}_{2}$ norm between the Doppler kernels. 


\section{B. Maxwell kernel \& Maxwell-over-velocity reconstruction derivations}

\section{B.1. Maxwell \&3 Maxwell-over-velocity kernels definition}

Physically, the Doppler broadening operation is defined through the conservation of the reaction rate in equation (1) as the relative energies span the Maxwellian distribution of velocities of the ideal gaz model for the target nucleus. When interpolating by linear combination of reference temperatures, this yields:

$$
\left(v \sigma_{T}^{(\text {True })}-v \sigma_{T}^{(\text {approx })}\right)(v)=\int_{v_{t} \mid v_{r}>0} v_{r} \sigma_{T_{0}}\left(v_{r}\right)\left[\mathcal{M}_{T}\left(\overrightarrow{v_{t}}\right)-\sum_{j} c_{j} \mathcal{M}_{T_{j}}\left(\overrightarrow{v_{t}}\right)\right] d \overrightarrow{v_{t}}
$$

Upon change of variables from vectorial velocity to scalar speeds, the Maxwell distribution is recovered:

$$
v \sigma_{T}(v)=\frac{1}{2} \int_{0}^{\infty}\left[v_{r} \sigma_{T_{0}}\left(v_{r}\right)\right] \frac{v_{r}}{v} d v_{r} \int_{\left|v-v_{r}\right|}^{\left|v+v_{r}\right|} \frac{\mathcal{M}_{T}\left(v_{t}\right)}{v_{t}} d v_{t}
$$

From this, we define the Maxwell kernel as:

$$
\mathcal{K}_{T}^{\mathbb{M}}: z \mapsto 4 s^{2} z^{2} \mathcal{K}_{T}^{\mathbb{B}}=4 z^{2} \frac{s^{3}}{\sqrt{\pi}} \mathrm{e}^{-s^{2} z^{2}}
$$

Using the definition $\xi=\frac{1}{4 s^{2}}$, the coefficients of the Gram matrix are then readily calculated

$$
\left\langle\mathcal{K}_{T_{i}}^{\mathbb{M}} \mid \mathcal{K}_{T_{j}}^{\mathbb{M}}\right\rangle=\frac{1}{4 \pi} \frac{1}{{\sqrt{\xi_{i} \xi_{j}}}^{3}} \int_{0}^{\infty} u^{4} e^{-u^{2}\left(\frac{1}{4 \xi_{i}}+\frac{1}{4 \xi_{j}}\right)} d u
$$

Defining the Gauss integrals of order $n$

$$
G_{n}=\int_{0}^{\infty} x^{n} e^{-x^{2}} d x
$$

and using the following recurrence formula

$$
\begin{aligned}
G_{n} & =\frac{n-1}{2} G_{n-2} \\
G_{1} & =\frac{1}{2} \\
G_{0} & =\frac{\sqrt{\pi}}{2}
\end{aligned}
$$

one finds

$$
\left\langle\mathcal{K}_{T_{i}}^{\mathbb{M}} \mid \mathcal{K}_{T_{j}}^{\mathbb{M}}\right\rangle=\frac{3}{\sqrt{\pi}} \frac{\xi_{i} \xi_{j}}{\left(\xi_{i}+\xi_{j}\right)^{5 / 2}}
$$

Let us note that equation (B.2) leaves the choice of reconstructing the $\frac{\mathcal{M}}{v}$ kernel instead of $\mathcal{M}$. In that particular case, the recurrence formula would have yielded the following Gram matrix coefficients:

$$
\left\langle\frac{\mathcal{K}_{T_{i}}^{\mathbb{M}}}{v} \mid \frac{\mathcal{K}_{T_{j}}^{\mathbb{M}}}{v}\right\rangle=\frac{1}{2 \sqrt{\pi}} \frac{1}{{\sqrt{\xi_{i}+\xi_{j}}}^{3}}
$$


B.1.1. Free Maxwell \& Maxwell-over-velocity kernels reconstruction

From the latter, the free Maxwell kernel reconstruction system is:

$$
\mathbb{M} \cdot C=Y
$$

with

$$
\left[\begin{array}{ccc}
\frac{T_{1} T_{1}}{\left(T_{1}+T_{1}\right)^{5 / 2}} & \cdots & \frac{T_{1} T_{N}}{\left(T_{1}+T_{N}\right)^{5 / 2}} \\
\vdots & \ddots & \vdots \\
\frac{T_{N} T_{1}}{\left(T_{N}+T_{1}\right)^{5 / 2}} & \cdots & \frac{T_{N} T_{N}}{\left(T_{N}+T_{N}\right)^{5 / 2}}
\end{array}\right] \cdot\left[\begin{array}{c}
c_{1} \\
\vdots \\
c_{N}
\end{array}\right]=\left[\begin{array}{l}
\frac{T_{1} T}{\left(T_{1}+T\right)^{5 / 2}} \\
\vdots \\
\frac{T_{N} T}{\left(T_{N}+T\right)^{5 / 2}}
\end{array}\right]
$$

If instead the choice of reconstructing $\frac{\mathcal{M}}{v}$ is made, then the $\mathrm{L}_{2}$ minimization solution is given by

$$
\frac{\mathbb{M}}{v} \cdot C=Y
$$

with

$$
\left[\begin{array}{ccc}
\frac{1}{{\sqrt{T_{1}+T_{1}}}^{3}} & \cdots & \frac{1}{{\sqrt{T_{1}+T_{N}}}^{3}} \\
\vdots & \ddots & \vdots \\
\frac{1}{{\sqrt{T_{N}+T_{1}}}^{3}} & \cdots & \frac{1}{{\sqrt{T_{N}+T_{N}}}^{3}}
\end{array}\right] \cdot\left[\begin{array}{c}
c_{1} \\
\vdots \\
c_{N}
\end{array}\right]=\left[\begin{array}{l}
\frac{1}{{\sqrt{T+T_{1}}}^{3}} \\
\vdots \\
\frac{1}{{\sqrt{T+T_{N}}}^{3}}
\end{array}\right]
$$

which is the Hadamard product $3^{\text {rd }}$ power of the Boltzmann kernel reconstruction problem.

\section{B.1.2. Constrained Maxwell \& Maxwell-over-velocity kernels reconstruction}

The constrained Maxwell kernel reconstruction problem is then

$$
\widetilde{\mathbb{M}} \cdot C=\widetilde{Y}
$$

where

$$
\left[\begin{array}{cccc}
\widetilde{m_{1,1}} & \cdots & \widehat{m_{1, N-1}} & 0 \\
\vdots & \ddots & \vdots & \vdots \\
\widetilde{m_{N-1,1}} & \cdots & \widehat{m_{N-1, N-1}} & 0 \\
1 & \cdots & 1 & 1
\end{array}\right] \cdot\left[\begin{array}{c}
c_{1} \\
\vdots \\
c_{N-1} \\
c_{N}
\end{array}\right]=\left[\begin{array}{l}
\widetilde{y_{1}} \\
\vdots \\
\widetilde{y_{N-1}} \\
1
\end{array}\right]
$$

with

$$
\widetilde{m_{i j}}=\frac{T_{i} T_{j}}{\left(T_{i}+T_{j}\right)^{5 / 2}}-\frac{T_{i} T_{N}}{\left(T_{i}+T_{N}\right)^{5 / 2}}-\frac{T_{N} T_{j}}{\left(T_{N}+T_{j}\right)^{5 / 2}}+\frac{T_{N} T_{N}}{\left(T_{N}+T_{N}\right)^{5 / 2}}
$$

and

$$
\widetilde{y_{i}}=\frac{T_{i} T}{\left(T_{i}+T\right)^{5 / 2}}-\frac{T_{i} T_{N}}{\left(T_{i}+T_{N}\right)^{5 / 2}}-\frac{T_{N} T}{\left(T_{N}+T\right)^{5 / 2}}+\frac{T_{N} T_{N}}{\left(T_{N}+T_{N}\right)^{5 / 2}}
$$

Again, the same considerations are true for the $\frac{\mathcal{M}}{v}$ reconstruction by replacing $\frac{T_{i} T_{j}}{\left(T_{i}+T_{j}\right)^{5 / 2}}$ by $\frac{1}{{\sqrt{T_{i}+T_{j}}}^{3}}$.

\section{Boltzmann kernel reconstruction derivations}

C.1. Casting the Doppler broadening operation as a convolution with the Boltzmann distribution

G. Ferran et al. [4] recently introduced a generalized, odd parity, function $s_{T}$ linked to the cross section as:

$$
s_{T}: E \mapsto\left\{\begin{aligned}
E \cdot \sigma_{T}(E), & \forall E \in \mathbb{R}_{+} \\
s_{T}(E)=-s_{T}(-E) & \forall E \in \mathbb{R}_{-}
\end{aligned}\right.
$$


Applying the Doppler broadening operation to $s_{T}$ yields a linear convolution product operator that transforms the generalized function $s_{0}$ from temperature $T_{0}$ to temperature $T>T_{0}$ as follows [4]:

$$
s_{T}=s_{0} \star \mathcal{K}_{T}^{\mathbb{B}}
$$

where the convolution product is defined as:

$$
\forall x \in \mathbb{R}, f \star g(x)=\int_{-\infty}^{\infty} f(t) g(x-t) d t
$$

and with $\mathcal{K}_{T}^{\mathbb{B}}$ representing the Boltzmann distribution of energies of the target nuclei.

$$
\mathcal{K}_{T}^{\mathbb{B}}:\left\{\begin{aligned}
\mathbb{R} & \rightarrow \mathbb{R}_{+} \\
t & \mapsto \frac{s}{\sqrt{\pi}} \mathrm{e}^{-s^{2} t^{2}}
\end{aligned}\right.
$$

The problem provides a Hilbert space structure through the $\mathrm{L}_{2}$ norm and its associated scalar product:

$$
\langle f \mid g\rangle=\int_{-\infty}^{\infty} f(t) g(t) d t
$$

Using $\xi_{i}=\frac{1}{4 s_{i}^{2}}$, it stems that the Gram matrix coefficients are:

$$
\left\langle\mathcal{K}_{T_{i}}^{\mathbb{B}} \mid \mathcal{K}_{T_{j}}^{\mathbb{B}}\right\rangle=\frac{1}{4 \pi \sqrt{\xi_{i} \cdot \xi_{j}}} \int_{-\infty}^{+\infty} \mathrm{e}^{-t^{2}\left[\frac{1}{4 \xi_{i}}+\frac{1}{4 \xi_{j}}\right]} d t
$$

and using the recurrence formula for the Gauss integrals (equations B.6), one finds:

$$
\left\langle\mathcal{K}_{T_{i}}^{\mathbb{B}} \mid \mathcal{K}_{T_{j}}^{\mathbb{B}}\right\rangle=\frac{1}{2 \sqrt{\pi\left(\xi_{i}+\xi_{j}\right)}}
$$

\section{C.2. Free Boltzmann kernel reconstruction}

The free Boltzmann kernel reconstruction solution for the $\mathrm{L}_{2}$ norm is thus found by inverting the system:

$$
\mathbb{B} \cdot C=Y
$$

with

$$
\left[\begin{array}{ccc}
\frac{1}{\sqrt{T_{1}+T_{1}}} & \cdots & \frac{1}{\sqrt{T_{1}+T_{N}}} \\
\vdots & \ddots & \vdots \\
\frac{1}{\sqrt{T_{N}+T_{1}}} & \cdots & \frac{1}{\sqrt{T_{N}+T_{N}}}
\end{array}\right] \cdot\left[\begin{array}{c}
c_{1} \\
\vdots \\
c_{N}
\end{array}\right]=\left[\begin{array}{l}
\frac{1}{\sqrt{T+T_{1}}} \\
\vdots \\
\frac{1}{\sqrt{T+T_{N}}}
\end{array}\right]
$$

\section{C.2.1. Constrained Boltzmann kernel reconstruction}

Similarly, the constrained Boltzmann kernel reconstruction problem is:

$$
\widetilde{\mathbb{B}} \cdot C=\widetilde{Y}
$$

where

$$
\left[\begin{array}{cccc}
\widetilde{b_{1,1}} & \cdots & \widetilde{b_{1, N-1}} & 0 \\
\vdots & \ddots & \vdots & \vdots \\
\widetilde{b_{N-1,1}} & \cdots & \widehat{b_{N-1, N-1}} & 0 \\
1 & \cdots & 1 & 1
\end{array}\right] \cdot\left[\begin{array}{c}
c_{1} \\
\vdots \\
c_{N-1} \\
c_{N}
\end{array}\right]=\left[\begin{array}{l}
\widetilde{y_{1}} \\
\vdots \\
\widetilde{y_{N-1}} \\
1
\end{array}\right]
$$


with

$$
\widetilde{b_{i j}}=\frac{1}{\sqrt{T_{i}+T_{j}}}-\frac{1}{\sqrt{T_{i}+T_{N}}}-\frac{1}{\sqrt{T_{N}+T_{j}}}+\frac{1}{\sqrt{T_{N}+T_{N}}}
$$

and

$$
\widetilde{y_{i}}=\frac{1}{\sqrt{T_{i}+T}}-\frac{1}{\sqrt{T_{i}+T_{N}}}-\frac{1}{\sqrt{T_{N}+T}}+\frac{1}{\sqrt{T_{N}+T_{N}}}
$$

\section{Physical justification of the choice of $\mathbf{L}_{2}$ norm for kernel reconstruction}

The performance of all these kernel reconstruction methods will be nuclide dependent, hence the choice in equation (13) of posing the problems as $\mathrm{L}_{2}$ norm minimizations may seem somewhat arbitrary. However, the general physical properties of cross sections tend to support the latter choice in the following way.

\section{D.1. $\mathrm{L}_{2}$ norm interpolation error for Boltzmann convolution Kernel}

When considering the linear combination temperature interpolation problem in the light of Ferran's convolution product approach, the difference between the exact generalized odd function $s_{T}$ and the interpolated one is given by:

$$
s_{T}^{(\text {True })}-s_{T}^{(\text {approx })}=s_{0} \star\left(\mathcal{K}_{T}^{\mathbb{B}}-\sum_{j=1}^{N} c_{j} \mathcal{K}_{T_{j}}^{\mathbb{B}}\right)
$$

Having the approximate temperature-interpolated cross section equating the true value (in a simple convergence sense) $\left(s_{T}^{(\text {True })}-s_{T}^{(\text {approx })}=0\right)$ is thus equivalent to verifying the condition:

$$
s_{0} \star\left(\mathcal{K}_{T}^{\mathbb{B}}-\sum_{j=1}^{N} c_{j} \mathcal{K}_{T_{j}}^{\mathbb{B}}\right)=0
$$

However, considerations hereafter exposed on $s_{0}$ show that $s_{0}$ is not of a compact support, which means the latter condition can be met if, and only if:

$$
\left(\mathcal{K}_{T}^{\mathbb{B}}-\sum_{j=1}^{N} c_{j} \mathcal{K}_{T_{j}}^{\mathbb{B}}\right)=0
$$

It is however not possible to fully satisfy such condition by interpolation, and thus a metric has to be chosen so as to minimize the distance among kernels to approach this condition. The $\mathrm{L}_{2}$ metric is a good choice in tune with the properties of $s_{0}$. Indeed, if it is sought to minimize the $\mathrm{L}_{2}$-distance between the two, this means finding the $\left(c_{j}\right)$ such as to minimize:

$$
\left\|s_{T}^{(\text {True })}-s_{T}^{(\text {approx })}\right\|_{L_{2}}=\left\|s_{0} \star\left(\mathcal{K}_{T}^{\mathbb{B}}-\sum_{j=1}^{N} c_{j} \mathcal{K}_{T_{j}}^{\mathbb{B}}\right)\right\|_{L_{2}}
$$

Recalling the Fourier transform property on the convolution product,

$$
\widehat{f \star g}=\widehat{f} \cdot \widehat{g}
$$

one can invoke the theorem of Parseval to establish that:

$$
\left\|s_{T}^{(\text {True })}-s_{T}^{(\text {approx })}\right\|_{L_{2}}=\left\|\widehat{s_{0}} \cdot\left(\widehat{\mathcal{K}_{T}^{\mathbb{B}}}-\sum_{j=1}^{N} c_{j} \widehat{\mathcal{K}_{T_{j}}^{\mathbb{B}}}\right)\right\|_{L_{2}}
$$




\section{D.2. Pole representation properties of nuclear cross sections}

To infer additional information, a careful analysis of the $\widehat{s_{0}}$ function is required. Angle-integrated nuclear cross sections can be cast into a sum of poles and residues according to the Pole Representation formalism [6] [7]. From which $s_{0}$ takes the general form:

$$
s_{0}(u)=s_{p o t}+\sum_{j} \Re\left[\frac{r_{j}}{p_{j}-u}\right]
$$

and thus the Fourier transform verify

$$
\widehat{s_{0}}(\nu)=\widehat{s_{p o t}}+\sum_{j} \widehat{\Re\left[\frac{r_{j}}{p_{j}-u}\right]}(\nu)
$$

The first term can be close to an inverse function or a sign function, the Fourier transforms of which are Fourier transforms of one-another. The poles will correspond to wide and flat Exponential integrals Fourier transforms, which can be derived analytically. On the contrary, the Boltzmann distributions will have Gaussian Fourier transforms. Frequency separation is thus a physically good approximation and one can treat $\widehat{s_{0}}$ as slowly varying in comparison to the Boltzmann kernels.

$$
\left\|\widehat{s_{0}} \cdot\left(\widehat{\mathcal{K}_{T}^{\mathbb{B}}}-\sum_{j=1}^{N} c_{j} \widehat{\mathcal{K}_{T_{j}}^{\mathbb{B}}}\right)\right\|_{L_{2}} \approx\left\|\widehat{s_{0}}\right\|_{L_{2}} \cdot\left\|\left(\widehat{\mathcal{K}_{T}^{\mathbb{B}}}-\sum_{j=1}^{N} c_{j} \widehat{\mathcal{K}_{T_{j}}^{\mathbb{B}}}\right)\right\|_{L_{2}}
$$

Thus, minimizing the $\mathrm{L}_{2}$ norm $\left\|s_{T}^{(\text {True })}-s_{T}^{(\text {approx })}\right\|_{\mathrm{L}_{2}}$ is close to minimizing the $\mathrm{L}_{2}$ distance between the Boltzmann energy distributions of the target particles.

\section{D.3. Quantifying cross section independent kernel reconstruction error}

How good an approximation the former is can be more accurately captured by further analysis, where the isotope specificity of each $s_{0}$ appears. Let $\mathcal{K}_{c}^{\mathbb{B}}=\left(\mathcal{K}_{T}^{\mathbb{B}}-\sum_{j=1}^{N} c_{j} \mathcal{K}_{T_{j}}^{\mathbb{B}}\right)$, and $c$ designate the vector of coefficients: $c=\left[c_{1}, \ldots, c_{j}, \ldots, c_{N}\right]^{\top}$

Bi-linearity of the hermitian product combined with the Plancherel-Parseval theorem then yield

$$
\begin{aligned}
\frac{\partial}{\partial c_{j}}\left\|s_{0} \star \mathcal{K}_{c}^{\mathbb{B}}\right\|_{L_{2}}^{2} & =\frac{\partial}{\partial c}\left\|\widehat{s_{0}} \cdot \widehat{\mathcal{K}_{c}^{\mathbb{B}}}\right\|_{L_{2}}^{2}=2 \Re\left[\left\langle\widehat{s_{0}} \cdot \widehat{\mathcal{K}_{c}^{\mathbb{B}}} \mid \widehat{s_{0}} \cdot \frac{\partial}{\partial c_{j}} \widehat{\mathcal{K}_{c}^{\mathbb{B}}}\right\rangle\right] \\
& =\Re\left[\left\langle{\widehat{s_{0}}}^{2} \mid \frac{\partial}{\partial c_{j}}\left({\widehat{\mathcal{K}_{c}^{\mathbb{B}}}}^{2}\right)\right\rangle\right]
\end{aligned}
$$

Or, in gradient notation:

$$
\nabla_{c}\left\|s_{0} \star \mathcal{K}_{c}^{\mathbb{B}}\right\|_{L_{2}}^{2}=\Re\left[\left\langle\left|{\widehat{s_{0}}}^{2}\right| \nabla_{c}\left(\widehat{\mathcal{K}}_{c}^{\mathbb{B}}\right)\right\rangle\right]
$$

Let us now consider the two optimal coefficient vectors, $c^{\left(s_{0}\right)}$ and $c^{(\mathcal{K})}$, which would minimize $\left\|s_{0} \star \mathcal{K}_{c}^{\mathbb{B}}\right\|_{\mathrm{L}_{2}}^{2}$ and $\left\|\mathcal{K}_{c}^{\mathbb{B}}\right\|_{L_{2}}^{2}$ respectively. Then, at the $c^{(\mathcal{K})}$ coefficients, which satisfy $\nabla_{c=c}(\mathcal{K})\left\|\mathcal{K}_{c}^{\mathbb{B}}\right\|_{L_{2}}^{2}=0$, the gradient becomes expressible as a function of the distance to the $\mathrm{L}_{2}$ mean:

$$
\nabla_{c=c}(\mathcal{K})\left\|s_{0} \star \mathcal{K}_{c}^{\mathbb{B}}\right\|_{\mathrm{L}_{2}}^{2}=\Re\left[\left\langle\left(\mid{\widehat{s_{0}}}^{2}-\|{\widehat{s_{0}}}_{\mathrm{L}_{2}}^{2}\right) \mid \nabla_{c=c}(\mathcal{K})\left(\widehat{\mathcal{K}}_{c}^{\mathbb{B}}\right)\right\rangle\right]
$$

Using the Hessian matrix

$$
\left[\nabla \nabla_{c=c}(\mathcal{K})\left\|s_{0} \star \mathcal{K}_{c}^{\mathbb{B}}\right\|_{L_{2}}^{2}\right]=\left[\Re\left[\left\langle{\widehat{s_{0}}}^{2} \mid \nabla \nabla_{c=c}(\mathcal{K})\left(\widehat{\mathcal{K}}_{c}^{\mathbb{B}}\right)\right\rangle\right]\right]
$$


to Taylor expand around $c^{(\mathcal{K})}$, and using the fact that $c^{\left(s_{0}\right)}$ satisfies $\nabla_{c=c^{\left(s_{0}\right)}}\left\|s_{0} \star \mathcal{K}_{c}^{\mathbb{B}}\right\|_{\mathrm{L}_{2}}^{2}=0$, the $1^{s t}$ order difference between the two optimal coefficients can be expressed as:

$$
\left(c^{\left(s_{0}\right)}-c^{(\mathcal{K})}\right)=-\left[\nabla \nabla_{c=c}(\mathcal{K})\left\|s_{0} \star \mathcal{K}_{c}^{\mathbb{B}}\right\|_{L_{2}}^{2}\right]^{-1}\left[\nabla_{c=c}(\mathcal{K})\left\|s_{0} \star \mathcal{K}_{c}^{\mathbb{B}}\right\|_{L_{2}}^{2}\right]+\mathcal{O}\left(\left\|c^{\left(s_{0}\right)}-c^{(\mathcal{K})}\right\|^{2}\right)
$$

from which it stems that:

$$
\begin{aligned}
\left\|s_{0} \star \mathcal{K}_{c=c^{\left(s_{0}\right)}}^{\mathbb{B}}\right\|_{\mathrm{L}_{2}}^{2}-\left\|s_{0} \star \mathcal{K}_{\left.c=c^{(}\right)}^{\mathbb{B}}\right\|_{\mathrm{L}_{2}}^{2}= & -\left[\nabla_{c=c}(\mathcal{K})\left\|s_{0} \star \mathcal{K}_{c}^{\mathbb{B}}\right\|_{\mathrm{L}_{2}}^{2}\right]^{\top}\left[\nabla \nabla_{c=c^{(\mathcal{K})}}\left\|s_{0} \star \mathcal{K}_{c}^{\mathbb{B}}\right\|_{\mathrm{L}_{2}}^{2}\right]^{-1}\left[\nabla_{c=c}(\mathcal{K})\left\|s_{0} \star \mathcal{K}_{c}^{\mathbb{B}}\right\|_{\mathrm{L}_{2}}^{2}\right] \\
& +\mathcal{O}\left(\left\|c^{\left(s_{0}\right)}-c^{(\mathcal{K})}\right\|^{2}\right)
\end{aligned}
$$

Thus, equation (D.14) enables us to estimate how close to the $s_{0}$ cross section dependent optimal linear interpolation coefficients, $c^{\left(s_{0}\right)}$, are the cross section independent optimal coefficients $c^{(\mathcal{K})}$ found by $\mathrm{L}_{2}$ kernel reconstruction; and equation (D.15) informs us on how far from cross section dependent $\mathrm{L}_{2}$ optimality the kernel reconstruction coefficients $c^{(\mathcal{K})}$ place us.

Thus, one can calculate for various cross sections the right-hand-side of equation (D.15) at their kernel reconstruction optimal $c^{(\mathcal{K})}$ and compare: the bigger the value, the most has been lost with respect to isotope-dependent optimality $c^{\left(s_{0}\right)}$ for this isotope. This quantifies the dependency in $s_{0}$ and reinforces the validity of the approximation provided the general mathematical properties of the Pole Representation of angle-integrated nuclear cross sections.

\section{E. Temperature optimized free Doppler kernel reconstruction reference temperatures $\left(T_{j}\right)$ distribution study}

Here are analyzed important properties of the temperature-optimized free Doppler kernel reconstruction reference temperature grid $\left(T_{j}\right)$.

\section{E.1. Structure of optimal temperature grid $\left(T_{j}\right) \&$ scaling properties}

The study of the optimal reference temperatures as a function of $N$ shows that the entire problem is scaled according to the dimensionless variable:

$$
\tau=\ln \left(\frac{T}{T_{\min }}\right) / \ln \left(\frac{T_{\max }}{T_{\min }}\right)
$$

and the entire system is determined only by the ratio $r=\left(\frac{T_{\max }}{T_{\min }}\right)$, i.e. for any $\left[T_{\min }, T_{\max }\right]$ with the same ratio $r=\left(\frac{T_{\max }}{T_{\min }}\right)$, the $\mathrm{L}_{2}$ relative error $\left(\frac{\Delta \epsilon}{\epsilon}\right)$ is left unchanged. Also, the optimal distribution is symmetric, centered on $\tau=1 / 2$. Thus,

$$
\left(\frac{\Delta \epsilon}{\epsilon}\right)_{\left(\tau_{i}\right)}(1-\tau)=\left(\frac{\Delta \epsilon}{\epsilon}\right)_{\left(\tau_{i}\right)}(\tau)=\left(\frac{\Delta \epsilon}{\epsilon}\right)_{\left(T_{i}\right)}(T) .
$$

A noteworthy property is that for the particular choice of $r=e$, where $e$ designates the Euler number, the optimal values match exactly those of the Chebyshev quadrature, as seen in figure (E.5). This hints that the optimization problem is some super-set quadrature, that can range from Chebyshev to Legendre according to the value of $r$. Indeed, the kernel reconstruction methods can be seen as a form of extended quadrature operation with separation of variables where the coefficients bear all the $T$ dependence and the energy $E$ dependence is carried out by the cross sections. 


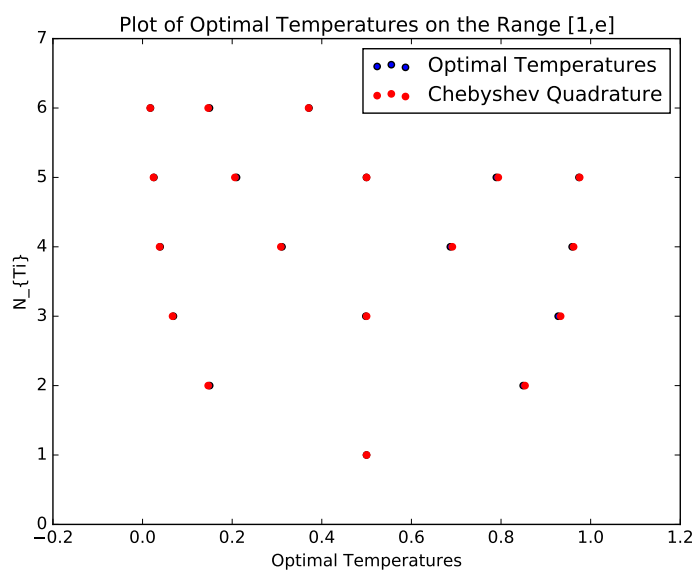

Figure E.5: Number of reference temperatures vs optimal temperatures on the range [1,e]. Optimal temperature points line up with Chebyshev quadrature.

\section{E.2. Optimal temperature grid for Doppler kernel reconstruction}

For the purpose of reactor analysis, the dimensionless $\tau_{i}$ solutions to the min-max optimization problem with ratio $r=10$ (which corresponds to the usual values used in nuclear reactor analysis) are hereafter reported in figure (E.6), and their scaled optimal values for $T_{\min }=300$ to $T_{\max }=3000$ reference temperatures corresponding values are recorded in table (E.1).
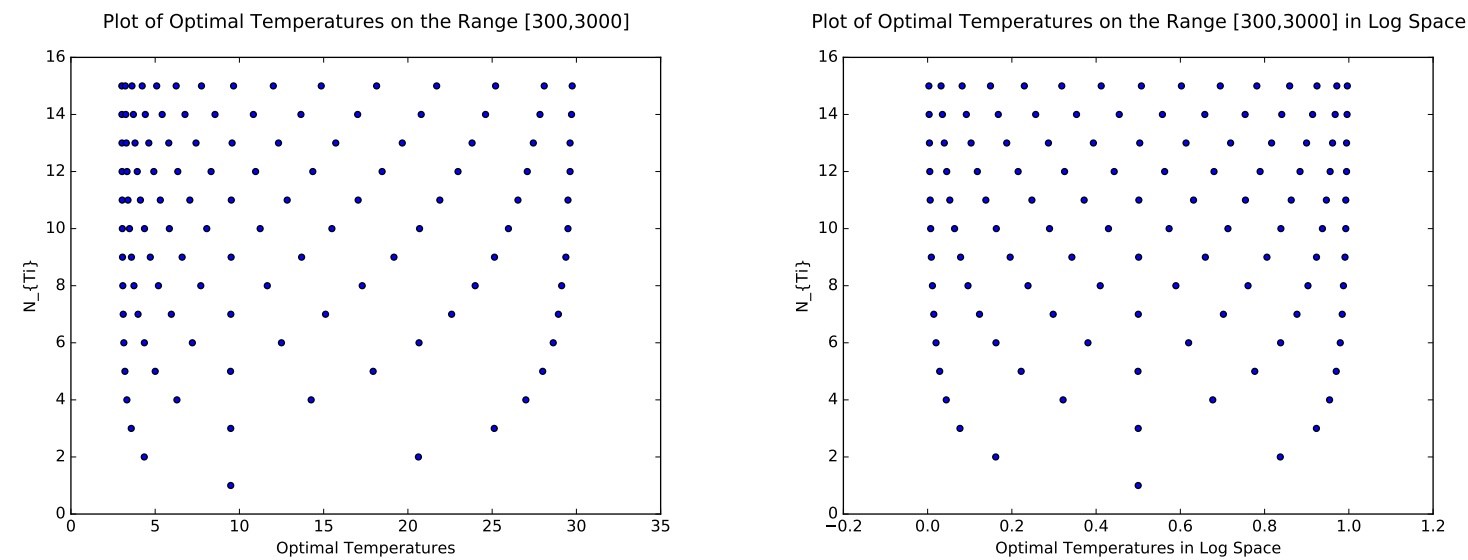

Figure E.6: Plots of number of reference temperatures vs optimal reference temperature grid for Doppler kernel reconstruction, in both linear and log space. In Linear space, the scaling properties are such that [3,30] is equivalent to $[300,3000]$, as the ratio $r=\left(\frac{T_{\max }}{T_{\min }}\right)$ is left unchanged.

\section{E.3. Temperature-optimized Doppler kernel reconstruction performance}

Here are assessed the efficiency gains in reconstructing the Doppler kernel as the grid of reference temperatures $\left(T_{j}\right)$ varies. These are quantified by comparing the maximum kernel reconstruction relative error $\left(\frac{\Delta \epsilon}{\epsilon}\right)_{\mathbb{D}}$ over the temperature range of interest $\left[T_{\min }, T_{\max }\right]$. The optimized temperature grid outperforms 


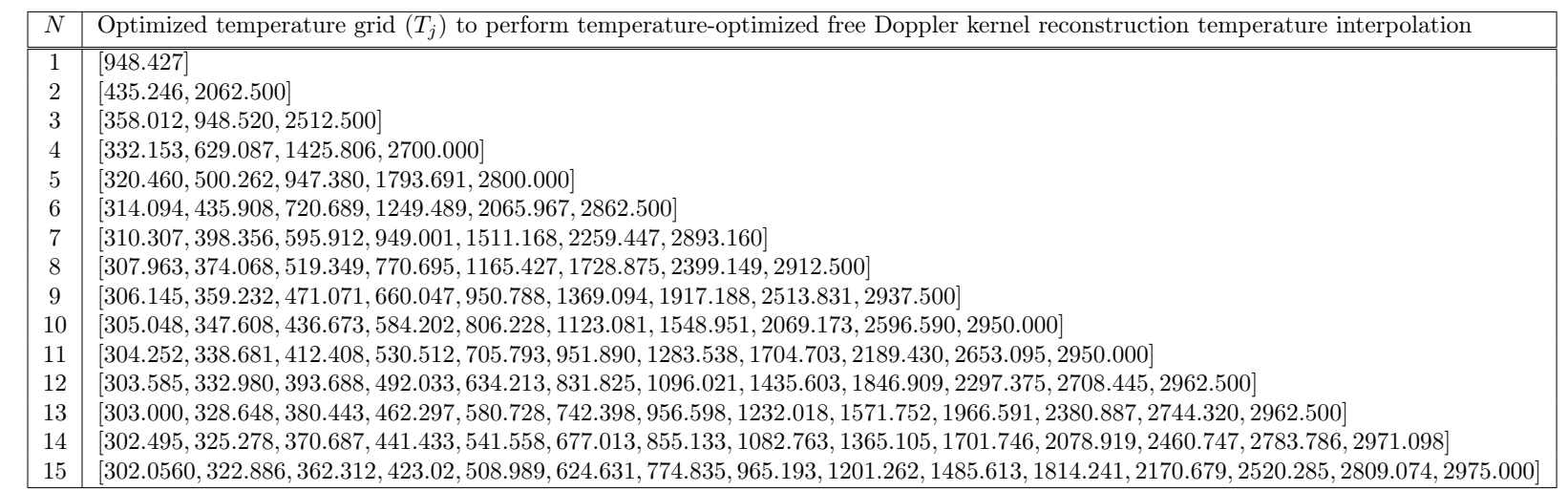

Table E.1: Optimal temperature grid for cross section interpolation using the free Doppler kernel reconstruction method on the range of temperatures [300,3000], for a given number $N$ of reference temperatures.

linear, square-root, and logarithmic spacing for any number of reference temperatures. As the number of reference temperatures increases, optimized spacing yields greater gains over other methods. This is illustrated in figure (E.7) and results are recorded in table (E.2) and plotted in (E.8).
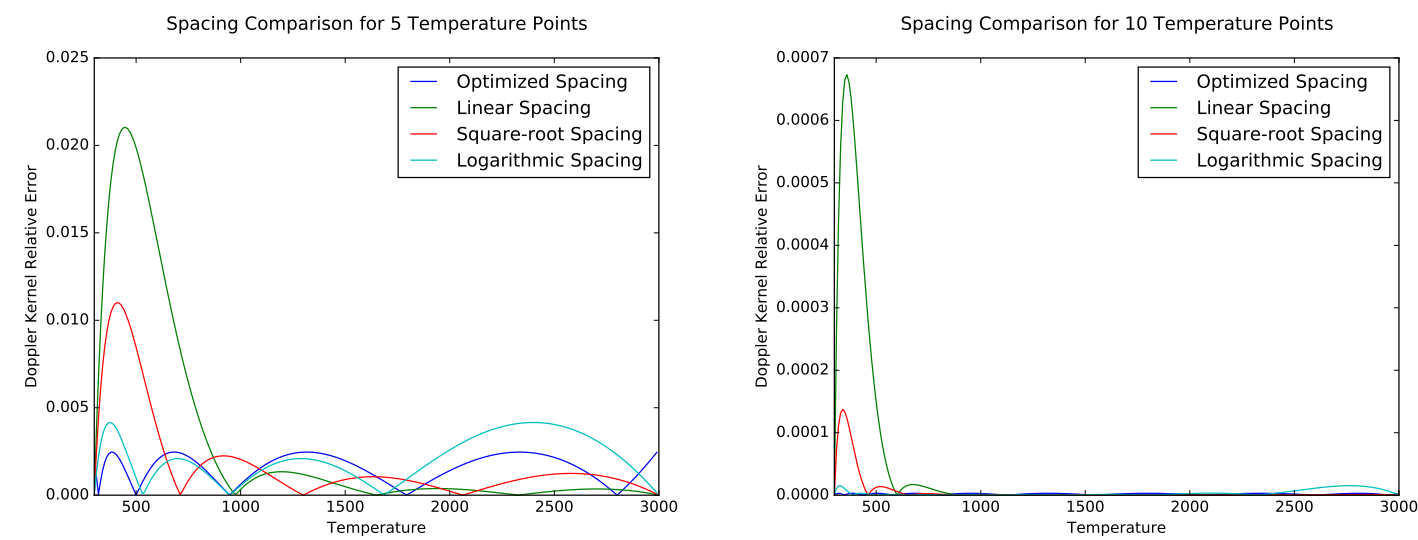

Figure E.7: Doppler kernel relative error over the range [300,3000] for both linearly spaced and optimized temperatures. 


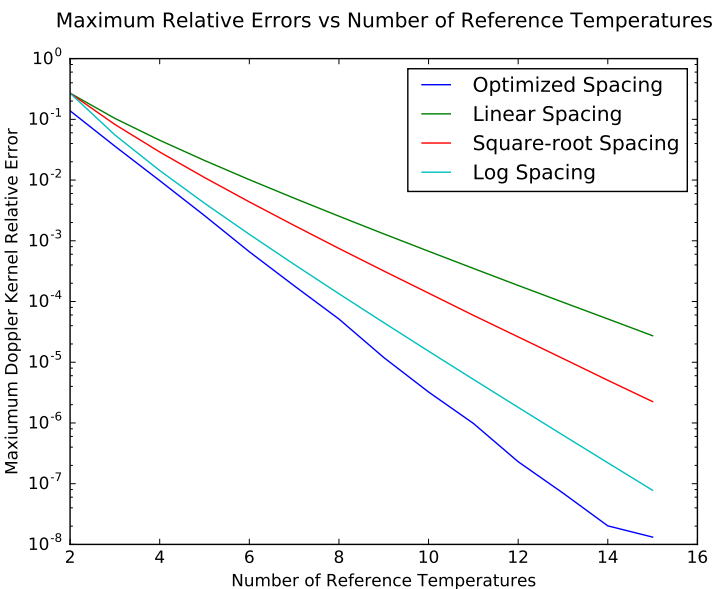

\begin{tabular}{|c|c|c|c|c|}
\hline$N$ & Optimized & Linear & Square-root & Logarithmic \\
\hline \hline 2 & $1.382 \times 10^{-1}$ & $2.698 \times 10^{-1}$ & $2.698 \times 10^{-1}$ & $2.698 \times 10^{-1}$ \\
3 & $3.614 \times 10^{-2}$ & $1.037 \times 10^{-1}$ & $8.241 \times 10^{-2}$ & $5.532 \times 10^{-2}$ \\
4 & $9.791 \times 10^{-3}$ & $4.524 \times 10^{-2}$ & $2.900 \times 10^{-2}$ & $1.435 \times 10^{-2}$ \\
5 & $2.600 \times 10^{-3}$ & $2.102 \times 10^{-2}$ & $1.101 \times 10^{-2}$ & $4.157 \times 10^{-3}$ \\
6 & $6.600 \times 10^{-4}$ & $1.015 \times 10^{-2}$ & $4.372 \times 10^{-3}$ & $1.279 \times 10^{-3}$ \\
7 & $1.819 \times 10^{-4}$ & $5.034 \times 10^{-3}$ & $1.791 \times 10^{-3}$ & $4.091 \times 10^{-4}$ \\
8 & $5.146 \times 10^{-5}$ & $2.542 \times 10^{-3}$ & $7.477 \times 10^{-4}$ & $1.342 \times 10^{-4}$ \\
9 & $1.197 \times 10^{-5}$ & $1.300 \times 10^{-3}$ & $3.184 \times 10^{-4}$ & $4.490 \times 10^{-5}$ \\
10 & $3.236 \times 10^{-6}$ & $6.731 \times 10^{-4}$ & $1.374 \times 10^{-4}$ & $1.522 \times 10^{-5}$ \\
11 & $9.833 \times 10^{-7}$ & $3.508 \times 10^{-4}$ & $5.923 \times 10^{-5}$ & $5.222 \times 10^{-6}$ \\
12 & $2.300 \times 10^{-7}$ & $1.839 \times 10^{-4}$ & $2.614 \times 10^{-5}$ & $1.807 \times 10^{-6}$ \\
13 & $7.005 \times 10^{-8}$ & $9.725 \times 10^{-5}$ & $1.151 \times 10^{-5}$ & $6.296 \times 10^{-7}$ \\
14 & $2.021 \times 10^{-8}$ & $5.166 \times 10^{-5}$ & $5.041 \times 10^{-6}$ & $2.208 \times 10^{-7}$ \\
15 & $1.319 \times 10^{-8}$ & $2.732 \times 10^{-5}$ & $2.247 \times 10^{-6}$ & $7.780 \times 10^{-8}$ \\
\hline
\end{tabular}

Figure E.8 \& Table E.2: Maximum relative error in reconstructing the Doppler kernel for a given number of reference temperatures $N$, for various temperature spacings. The optimal reference temperatures grid $\left(T_{j}\right)$ significantly outperforms all other reference temperature grid spacing.

\section{REFERENCES}

[1] T. Trumbull, Treatment of nuclear data for transport problems containing detailed temperature distributions, Nuclear Technology 156 (2006) 75-86.

[2] S. Peng, A. B. S. Zhang, X. Jiang, Investigations on the point-wise neutron cross-section temperature interpolation methods, Annals of Nuclear Energy 45 (2012) 155-160.

[3] D. Cullen, C. Weisbin, Exact doppler broadening of tabulated cross sections., Nucl. Sci. Eng. 60 (3).

[4] G. Ferran, M. Gonin, A new method for the doppler broadening of the solbrig's kernel using a fourier transform, Nucl. Sci. Eng. 179 (2015) 285-301.

[5] W. R. Martin, S. Wilderman, F. B. Brown, G. Yesilyurt, Implementation of on-the-fly doppler broadening in mcnp, in: International Conference on Mathematics and Computational Methods Applied to Nuclear Science and Engineering, Sun Valley, Idaho, 2013.

[6] R. N. Hwang, A rigorous pole representation of multilevel cross sections and its practical applications, Nucl. Sci. Eng. 96 (1987) 192-209.

[7] C. Josey, P. Ducru, B. Forget, K. Smith, Windowed multipole for cross section doppler broadening, Journal of Computational Physics 307 (2016) 715-727. 